\title{
The Genetic and Epigenetic Mechanisms Involved in Irreversible Pulp Neural Inflammation
}

\author{
Xiaoxi Xi $\mathbb{D}^{1},{ }^{1}$ Yihong Ma $\mathbb{D},{ }^{2}$ Yuzhen Xu $\mathbb{D},{ }^{3}$ Anthony Chukwunonso Ogbuehi $\mathbb{D},{ }^{4}$ \\ Xiangqiong Liu $\mathbb{D}^{5}{ }^{5}$ Yupei Deng $\mathbb{D}^{5}{ }^{5}$ Junming Xi $\mathbb{D}^{1},{ }^{1}$ Haitong Pan $\mathbb{D}^{6},{ }^{6}$ Qian Lin $\mathbb{D}^{7},{ }^{7}$ Bo Li $\mathbb{D}^{8}$, \\ Wanchen Ning $\mathbb{D},{ }^{9}$ Xiao Jiang $\mathbb{D},{ }^{10}$ Hanluo $\mathrm{Li} \mathbb{D},{ }^{11}$ Simin $\mathrm{Li} \mathbb{D},{ }^{10}$ and Xianda $\mathrm{Hu} \mathbb{D}^{5}$ \\ ${ }^{1}$ Department of Stomatology, Northeast Petroleum University Affiliated Hospital, Fazhan Road, High Tech District, \\ 163000 Daqing City, Heilongjiang Province, China \\ ${ }^{2}$ Department of Neurology, Graduate School of Medical Sciences, Kumamoto University, Kumamoto 860-0811, Japan \\ ${ }^{3}$ Department of Neurology, Shanghai Tenth People's Hospital, Tongji University School of Medicine, No. 301 Middle Yanchang Road, \\ Shanghai, China \\ ${ }^{4}$ Department of Physics, University of Münster, Wilhelm-Klemm-Str. 9, 48149 Münster, Germany \\ ${ }^{5}$ Laboratory of Molecular Cell Biology, Beijing Tibetan Hospital, China Tibetology Research Center, 218 Anwaixiaoguanbeili Street, \\ Chaoyang, Beijing 100029, China \\ ${ }^{6}$ Department of Stomatology, Daqing Oilfield General Hospital, Zhongkang Street No. 9, Saertu District, 163000 Daqing City, \\ Heilongjiang Province, China \\ ${ }^{7}$ Department of Prosthetics, School of Stomatology, Second Affiliated Dental Hospital of Jiamusi University, Hongqi Street No. 522, \\ Jiamusi City, Heilongjiang Province, China \\ ${ }^{8}$ Department of Stomatology, South District Hospital, Daqing Oilfield General Hospital Group, Tuqiang Fourth Street No. 14, \\ Hong Gang District, Daqing City, Heilongjiang Province, China \\ ${ }^{9}$ Department of Conservative Dentistry and Periodontology, Ludwig-Maximilians-University of Munich, Goethestrasse 70, \\ 80336 Munich, Germany \\ ${ }^{10}$ Stomatological Hospital, Southern Medical University, 510280 Guangzhou, China \\ ${ }^{11}$ Department of Cranio Maxillofacial Surgery, University Clinic Leipzig, Liebigstr. 12, 04103 Leipzig, Germany
}

Correspondence should be addressed to Xianda Hu; hellocean@hotmail.com

Received 9 September 2020; Revised 2 November 2020; Accepted 13 January 2021; Published 9 March 2021

Academic Editor: Wen-Jun Tu

Copyright ( 2021 Xiaoxi Xi et al. This is an open access article distributed under the Creative Commons Attribution License, which permits unrestricted use, distribution, and reproduction in any medium, provided the original work is properly cited.

Aim. To identify the critical genetic and epigenetic biomarkers by constructing the long noncoding RNA- (lncRNA-) related competing endogenous RNA (ceRNA) network involved in irreversible pulp neural inflammation (pulpitis). Materials and Methods. The public datasets regarding irreversible pulpitis were downloaded from the gene expression omnibus (GEO) database. The differential expression analysis was performed to identify the differentially expressed genes (DEGs) and DElncRNAs. Functional enrichment analysis was performed to explore the biological processes and signaling pathways enriched by DEGs. By performing a weighted gene coexpression network analysis (WGCNA), the significant gene modules in each dataset were identified. Most importantly, DElncRNA-DEmRNA regulatory network and DElncRNA-associated ceRNA network were constructed. A transcription factor- (TF-) DEmRNA network was built to identify the critical TFs involved in pulpitis. Result. Two datasets (GSE92681 and GSE77459) were selected for analysis. DEGs involved in pulpitis were significantly enriched in seven signaling pathways (i.e., NOD-like receptor (NLR), Toll-like receptor (TLR), NF-kappa B, tumor necrosis factor (TNF), cell adhesion molecules (CAMs), chemokine, and cytokine-cytokine receptor interaction pathways). The ceRNA regulatory relationships were established consisting of three genes (i.e., LCP1, EZH2, and NR4A1), five miRNAs (i.e., miR-3405p, miR-4731-5p, miR-27a-3p, miR-34a-5p, and miR-766-5p), and three lncRNAs (i.e., XIST, MIR155HG, and LINC00630). Six transcription factors (i.e., GATA2, ETS1, FOXP3, STAT1, FOS, and JUN) were identified to play pivotal roles in pulpitis. 
Conclusion. This paper demonstrates the genetic and epigenetic mechanisms of irreversible pulpitis by revealing the ceRNA network. The biomarkers identified could provide research direction for the application of genetically modified stem cells in endodontic regeneration.

\section{Introduction}

Pulpitis, as the neuroinflammation of the sensory trigeminal afferent axons in the dental pulp tissue, is accompanied by the pain induced by the stimulation of the pulp nerve fibers [1]. As a dynamic immune-inflammatory disease, the balance between the inflammatory and regenerative responses in the diseased pulp determines the clinical outcome, for example, from healthy pulp to reversible pulpitis, to irreversible pulpitis, and until pulp necrosis and pulp death [2]. Irreversible pulpitis is of high therapeutical relevance, as it is characterized by lingering pain that is featured by thermal stimuli, spontaneous pain, and pain at night [3]. Root canal therapy (RCT) based on pulpotomy remains the only choice for irreversible pulpitis; however, RCT can cause the teeth to be more brittle and thus more easily fractured [4]. Given this complication of RCT, researchers are attempting to use a combination of mesenchymal stem cells, biomaterial scaffolds, and growth factors to preserve dental pulp and achieve the neurovascularization of pulp tissue based on methods of modern tissue engineering. Nevertheless, pulp regeneration approaches face many challenges such as lifespan and diffusion of growth factor, as well as degradation of biomaterial. To overcome all of these challenges, genetically modified stem cells have been increasingly investigated and have also been shown to achieve better efficacy compared to using stem cells alone [5]. Since genetically modified stem cell transplantation could be promising in endodontic regeneration, it is therefore essential to have a deep understanding of the genetic and epigenetic mechanisms involved in the pathology of pulpitis.

With the advent of gene detection techniques, the genetic and epigenetic mechanisms have been shown by microarray and sequencing datasets $[6,7]$ to play a critical role in the immune-inflammatory response and repair response of pulpitis. As is well known, a messenger RNA (mRNA) as a protein-coding RNA can be targeted by multiple noncoding RNAs such as microRNAs (miRNAs) and long noncoding RNAs (lncRNAs) $[8,9]$. Based on the competing endogenous RNA (ceRNA) hypothesis proposed by Salmena et al., lncRNAs harboring miRNA response elements (MREs) and mRNAs can compete with each other by binding to a shared miRNA, thereby acting as molecular "sponges" and inducing translational repression at the posttranscriptional level [10]. Since the ceRNA network has been demonstrated to be involved in many inflammatory conditions and cancers [11], this network is possibly also involved in pulpal inflammation and should therefore be investigated. The bioinformatic techniques integrate all of the expression profiling datasets available to the public and allow the identification of critical biomarkers involved in the ceRNA network to be possible. Up until now, there is only one study available that examined this issue; however, there are differences in terms of study designs and processes, thereby obtaining quite distinct results [12].
Therefore, the current study is aimed at identifying many genetic and epigenetic biomarkers, including significantly enriched pathways of differentially expressed genes, critical genes involved in the protein-protein interaction network and the ceRNA network, critical miRNAs and lncRNAs involved in the ceRNA network, and transcription factors involved in the TF-mRNA network. The identification of these biomarkers will be helpful for the genetic modification of stem cells and will benefit pulpal regeneration and the shift from irreversible pulpitis to reversible pulpitis.

\section{Methods}

2.1. Procurement of Datasets. The microarray datasets regarding irreversible pulpitis were searched from the GEO of the NCBI database [13]. The genetic datasets could be investigating mRNA expression profile or noncoding RNA expression profile. The inclusion criteria of datasets were established as follows. (1) The study design of the included datasets should be established as two groups, including normal pulp tissue as the control group and inflamed pulp tissue as the experimental group. (2) The samples were taken from the adults (18 years or older) presenting for endodontic treatment with no evidence of periapical pathoses (i.e., radiolucency, swelling, and pressure sensitivity) and no previous pulp therapy (i.e., pulp capping). (3) Normal pulp tissues in the control group of the included datasets were collected from healthy third molars or teeth extracted for orthodontic purpose. Inflamed pulp tissues in the experimental group of the included datasets were extracted from teeth diagnosed with irreversible pulpitis in accordance with the endodontics diagnoses system from the American Association of Endodontists. (4) The sample size for each group in the included datasets should be more than three. The exclusion criteria of datasets were established as follows: (1) the datasets which had the different study design; (2) the datasets which took the pulp samples from the teeth with periodontitis/incompletely developed roots; (3) the datasets which took the pulp samples from the patients who had a compromised immune system or those who were taking medications known to influence the immune response; (4) the sample size for each group in the included datasets was less than three. According to the inclusion and exclusion criteria mentioned above, two datasets (GSE92681 and GSE77459) were therefore obtained.

2.2. Procurement of miRNA-mRNA and miRNA-lncRNA Interaction Data. The human's experimentally validated miRNA-target interaction pairs' data that have been validated by experiments were downloaded from three databases: TarBase (version 6.0) [14], miRTarBase (version 4.5) [15], and miRecords (version 4) [16]. The human's experimentally validated miRNA-lncRNA interaction pairs' data were downloaded from the starBase (version 2.0) database [17]. 


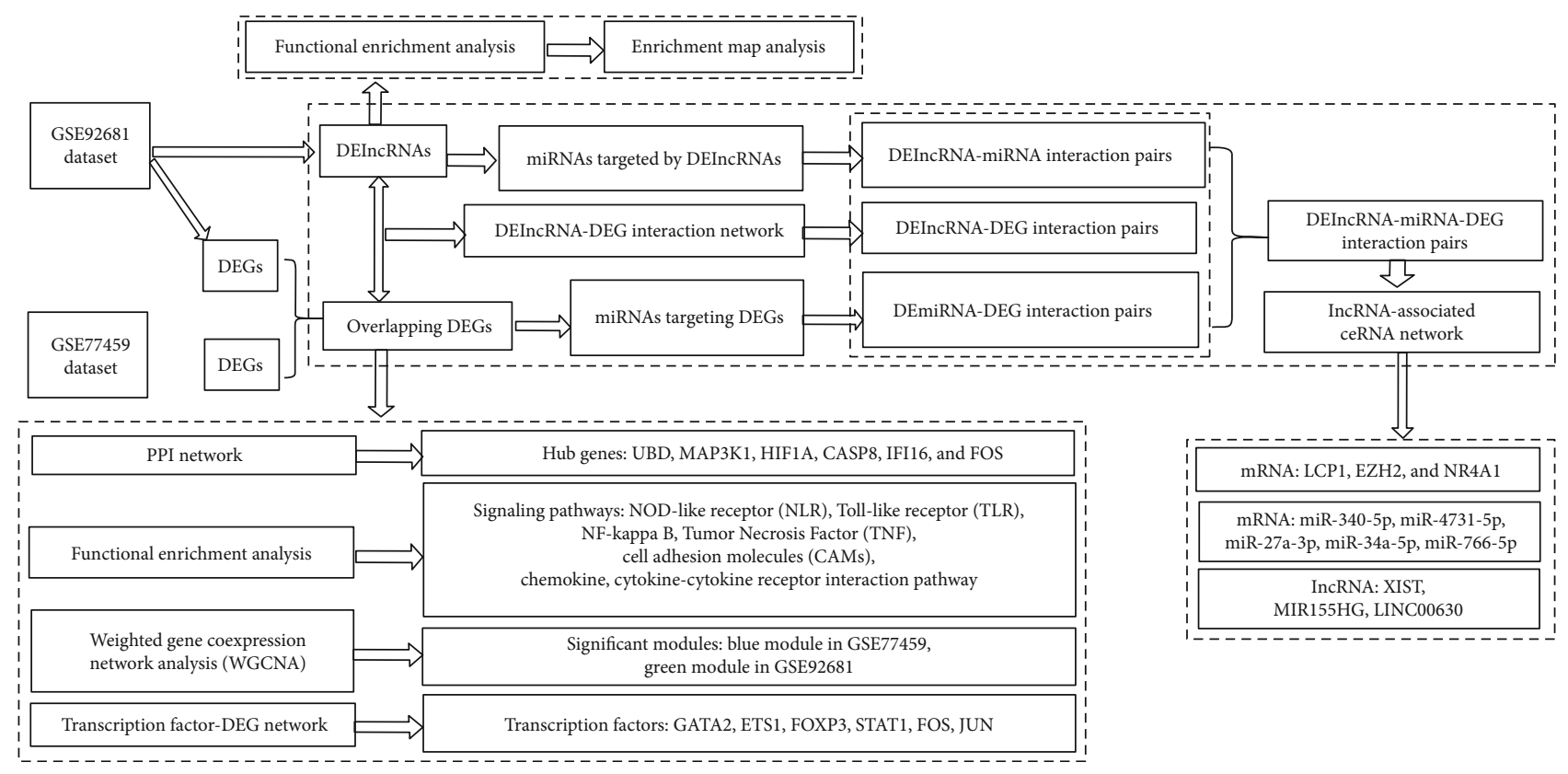

FIGURE 1: The flowchart of the present study. Two datasets (GSE92681 and GSE77459) were analyzed in this research by using varying bioinformatic analyzing methods, for example, differential expression analysis, functional enrichment analysis, weighted gene coexpression network analysis (WGCNA), enrichment map analysis, and network construction analysis (e.g., TF-DEG network, DElncRNA-DEG network, and ceRNA network).

2.3. Differential Expression Analysis. Regarding the dataset GSE92681, the probe sequences were reannotated because the corresponding gene symbols of probes cannot be obtained. The lncRNA and mRNA data were obtained from the platform of the GSE92681 dataset after annotation. The differential expression analysis was performed by using the Linear Models for Microarray data (limma) R/Bioconductor package [18] to identify the differentially expressed genes (DEGs), and differentially expressed lncRNAs (DElncRNAs) were identified between irreversible pulpitis samples and control healthy pulpal samples. The gene and lncRNAs that had the required cutoff criteria ( $p$ value $<0.05$ and a $\mid \log _{2}$ fold change (FC) $\mid>1$ ) were considered as DEGs and DElncRNAs. The Venn diagram was used to visualize the overlapped and union DEGs identified by two datasets.

2.4. Functional Enrichment Analysis of DEGs. The DEGs overlapped by two datasets (GSE77459 and GSE92681) were used for the functional enrichment analysis in order to explore the regulated biological processes and signaling pathways that are involved by these DEGs. The functional enrichment analysis was performed by using clusterProfiler package in the Bioconductor package [19]. The functional terms with $p$ value $<0.05$ were regarded to be significant.

2.5. Construction of Protein-Protein Interaction (PPI) Network. To comprehensively analyze the functions of DEGs involved in the entire biological network of pulpitis, the union DEGs identified from two datasets were used for the PPI network analysis instead of only taking the overlapped intersection DEGs. The interacting genes of these DEGs were downloaded from HPRD [20] and the BioGRID database
TABLE 1: The pulpitis-related datasets used for the present analysis.

\begin{tabular}{lccc}
\hline Datasets & $\begin{array}{c}\text { Experimental } \\
\text { type of datasets }\end{array}$ & $\begin{array}{c}\text { Sample size of } \\
\text { inflamed pulp } \\
\text { tissue }\end{array}$ & $\begin{array}{c}\text { Sample size of } \\
\text { healthy pulp } \\
\text { tissue }\end{array}$ \\
\hline GSE92681 & $\begin{array}{c}\text { Noncoding } \\
\text { RNA }\end{array}$ & 7 & 5 \\
GSE77459 & mRNA & 6 & 6 \\
\hline
\end{tabular}

TABLE 2: The number of upregulated and downregulated DEGs or DElncRNAs identified from included datasets.

\begin{tabular}{lccc}
\hline Datasets & $\begin{array}{c}\text { Number of } \\
\text { upregulated } \\
\text { factors }\end{array}$ & $\begin{array}{c}\text { Number of } \\
\text { downregulated } \\
\text { factors }\end{array}$ & $\begin{array}{c}\text { Number of } \\
\text { total factors }\end{array}$ \\
\hline $\begin{array}{l}\text { GSE92681_ } \\
\text { DElncRNA }\end{array}$ & 138 & 136 & 274 \\
$\begin{array}{l}\text { GSE92681_ } \\
\text { DEG }\end{array}$ & 486 & 178 & 664 \\
$\begin{array}{l}\text { GSE77459_ } \\
\text { DEG }\end{array}$ & 823 & 278 & 1101 \\
\hline
\end{tabular}

[21]. The visualization of a PPI network was performed by using Cytoscape software [22]. Several topological features (i.e., degree, average shortest path length, betweenness centrality, closeness centrality, clustering coefficient, and topological coefficient) of the nodes (protein) in this PPI network were calculated by using CytoNCA plugin in Cytoscape software to screen hub genes. The top 20 genes were selected from this network, and their topological features were listed. 


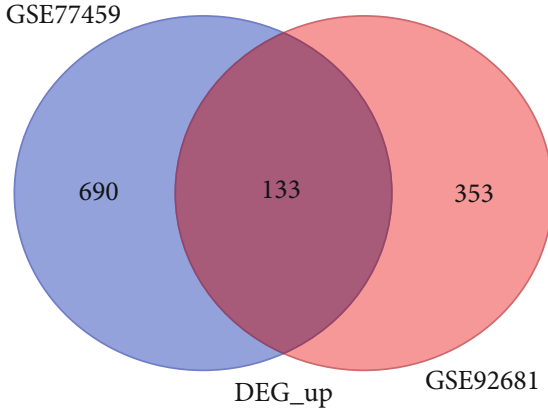

(a)

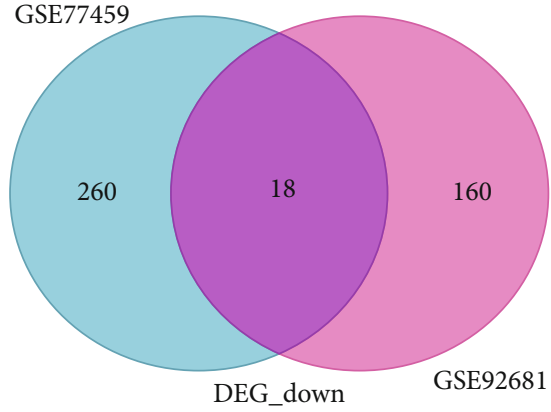

(b)

FIGURE 2: The Venn diagram shows the (a) up- and (b) downregulated DEGs identified by two datasets (GSE77459 and GSE92681). 133 upregulated DEGs and 18 downregulated DEGs were found to be overlapped between DEGs of GSE77459 and GSE92681.

2.6. Weighted Gene Coexpression Network Analysis. To further analyze the functions of interacting genes in the pathogenesis of pulpitis, the weighted gene coexpression network was constructed by using weighted gene coexpression network analysis (WGCNA). The genes with $p$ value $<0.05$ were selected, and the expression profile data of these genes were obtained. The significant gene modules were selected, and genetic interactions within each module were investigated. Based on the HPRD and BioGRID database, the PPI network of the selected significant gene modules was constructed, respectively. The top 25 gene nodes in these PPI networks were calculated and listed.

2.7. Functional Enrichment Analysis of DElncRNAs. Based on the GSE92681 dataset, the coexpression status of DElncRNAs and DEGs was calculated by using a statistical method-Pearson correlation. The significant interaction pairs with PPC (Pearson correlation coefficient) $>0.98$ and $p$ value $<0.05$ were selected. The functional enrichment analysis using clusterProfiler was performed to investigate the function of DElncRNAs within the selected significant interaction pairs. The function terms with $p$ value $<0.05$ were regarded as significant function terms.

2.8. Enrichment Map Analysis. The enrichment map analysis using Cytoscape plugin was performed for functional enrichment visualization. The enrichment map organizes enriched terms into a network with edges connecting overlapping gene sets [23]. This map in the present study was constructed to show the similarity among the function terms of genes regulated by lncRNAs. The enriched functions of genes targeted by DElncRNAs can interact with each other instead of being separate and isolated; thus, the dysregulation of a certain function term may result in the aberrant regulation of its interacted functions terms. In the enrichment map, nodes represent the functional GO terms. The color intensity of nodes represents significance ( $p$ value), and the color of nodes is lighter when the $p$ value is bigger. The edge thickness represents the degree of gene overlap that exists between two GO terms. The edge is wider when the mutual gene overlap between two GO terms is larger; that is to say, the similarity between these two GO terms is bigger.
2.9. Construction of a DElncRNA-DEG Regulatory Network. The interaction pairs of DElncRNA-DEG were obtained and used to construct a DElncRNA-DEG regulatory network. The topological characteristics of nodes in this network were calculated, and the top 20 nodes were ranked in descending order according to the degree.

2.10. Construction of a Transcription Factor- (TF-) DEG Network. First, DEGs obtained from two datasets (GSE92681 and GSE77459) were combined and used for subsequent analysis. The transcription factor- (TF-) DEG interaction pairs were then obtained from several databases, including TRANSFAC [24], TRED [25], and ORTI [26]. Based on these interaction pairs, the TF-DEG regulatory network was constructed. The topological feature of the nodes in this TF-DEG network was calculated, and the top 20 nodes were ranked in descending order according to the degree.

2.11. Construction of a ceRNA Network. The miRNAs that target DEGs and miRNAs targeted by DElncRNAs were obtained from the starBase database [17]. Afterward, we integrated coexpressed DElncRNA-mRNA interaction pairs, DElncRNA-miRNA interaction pairs, and DEG-miRNA interaction pairs. Based on these interaction pairs, a ceRNA network was constructed consisting of DElncRNA-miRNADEmRNA interaction pairs. The topological feature of nodes in this ceRNA network was calculated, and the top 20 nodes were listed in a descending rank according to the degree. In addition, in order to obtain the functional modules between lncRNA-mRNA interactions and miRNA-mRNA interactions, the Cytoscape plugin MCODE is used to identify the clusters in the ceRNA network.

\section{Results}

3.1. The Study Flowchart. The analyzing sequence of the present study is presented in Figure 1. As shown in Figure 1, two datasets regarding irreversible pulpitis were analyzed by performing differential expression analysis to identify DEGs and DElncRNAs, by carrying out functional enrichment analysis to identify signaling pathways, by constructing the DEG-TF network to identify critical TFs, and finally by building 


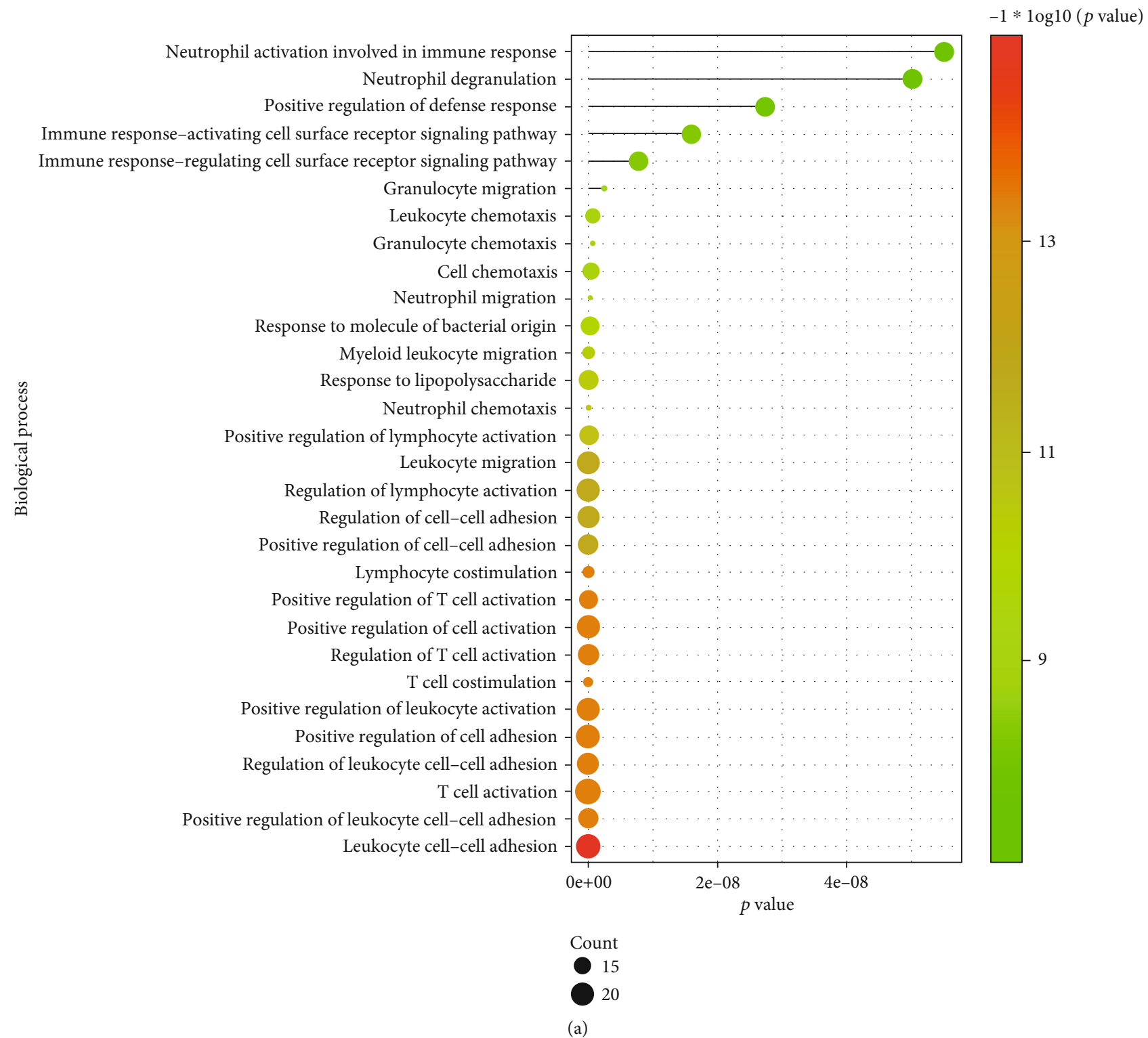

Figure 3: Continued. 


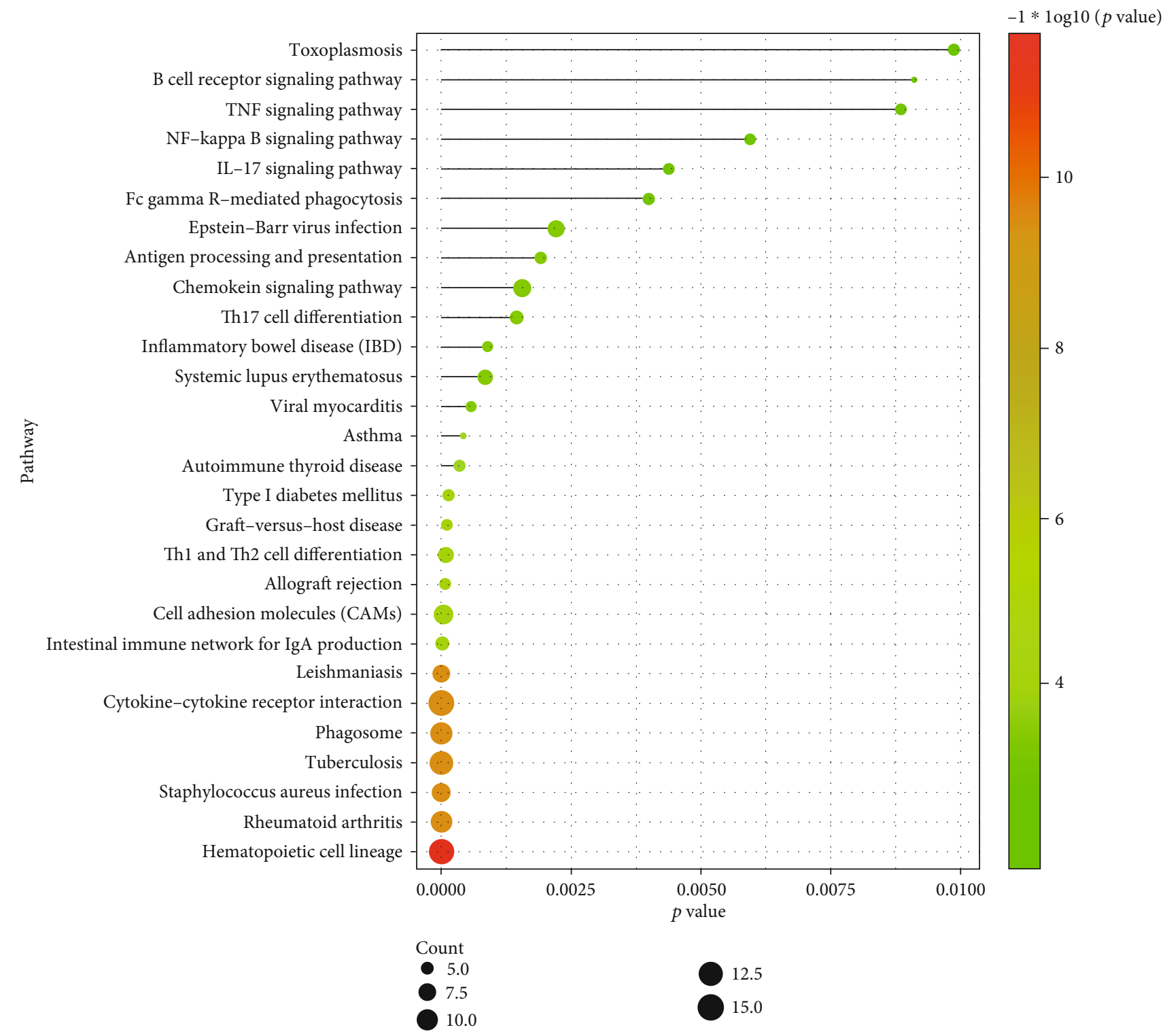

(b)

FIGURE 3: The functional enrichment analysis of DEGs overlapped in two datasets (GSE77459 and GSE92681). (a) The significantly enriched biological processes of overlapped DEGs. (b) The significantly enriched signaling pathways of overlapped DEGs.

lncRNA-associated ceRNA network to identify the critical genes, miRNAs, and lncRNAs.

3.2. Identification of DEGs. The GSE92681 dataset based on the GPL16956 platform analyzed the noncoding RNA expression profiling data of 7 inflamed pulpal tissues and 5 healthy pulpal tissues (Table 1). The GSE77459 dataset based on the GPL17692 platform analyzed the mRNA expression profiling data of 6 inflamed pulpal tissues and 6 healthy pulpal tissues (Table 1). From the dataset GSE92681, 274 DElncRNAs (138 upregulated and 136 downregulated) and 664 DEGs (486 upregulated and 178 downregulated) were identified (Table 2). From the dataset GSE77459, a total of 1,101 DEGs consisting of 823 upregulated and 278 downregulated were identified (Table 2). In addition, the intersection parts shown in the Venn diagram (Figure 2) show that 151 DEGs including 133 upregulated DEGs and 18 downregulated DEGs were found to be overlapped by two datasets. When considering the union parts of the Venn diagram, a total of 1,176 upregulated DEGs and 438 downregulated DEGs were identified.

3.3. Biological Processes and Signaling Pathways Enriched by DEGs. As shown in Figure 3(a), DEGs were significantly involved in many biological processes, for instance, cell adhesion-related BPs, immune cells (e.g., 3T cell, neutrophil, granulocyte, leukocyte, and lymphocyte)-related BPs, and immune response-related BPs. As shown in Figure 3(b), DEGs were significantly involved in many signaling 
TABLE 3: The topological characteristics of the top 20 nodes in the PPI network.

\begin{tabular}{|c|c|c|c|c|c|c|}
\hline $\begin{array}{l}\text { Gene } \\
\text { name }\end{array}$ & Degree & $\begin{array}{c}\text { Average shortest path } \\
\text { length }\end{array}$ & $\begin{array}{c}\text { Betweenness } \\
\text { centrality }\end{array}$ & $\begin{array}{l}\text { Closeness } \\
\text { centrality }\end{array}$ & $\begin{array}{l}\text { Clustering } \\
\text { coefficient }\end{array}$ & $\begin{array}{c}\text { Topological } \\
\text { coefficient }\end{array}$ \\
\hline UBD & 656 & 2.700089 & 0.092136 & 0.370358 & 0.001564 & 0.003702 \\
\hline UBC & 460 & 2.20363 & 0.309699 & 0.453797 & 0.004092 & 0.005551 \\
\hline IFI16 & 436 & 2.853032 & 0.052658 & 0.350504 & 0.001381 & 0.005531 \\
\hline ARRB2 & 369 & 2.872067 & 0.040035 & 0.348181 & 0.001915 & 0.007365 \\
\hline HLA-B & 338 & 2.825255 & 0.037735 & 0.35395 & 0.002915 & 0.00551 \\
\hline $\mathrm{EZH} 2$ & 301 & 2.891545 & 0.030774 & 0.345836 & 0.00206 & 0.008083 \\
\hline SFN & 296 & 2.903608 & 0.032059 & 0.344399 & 0.00213 & 0.00725 \\
\hline ADRB2 & 294 & 2.855246 & 0.033685 & 0.350233 & 0.003088 & 0.006755 \\
\hline LYN & 243 & 2.795595 & 0.03365 & 0.357706 & 0.013332 & 0.008931 \\
\hline MAP3K1 & 229 & 2.901173 & 0.021399 & 0.344688 & 0.00406 & 0.009621 \\
\hline FOS & 226 & 2.943448 & 0.02579 & 0.339738 & 0.00468 & 0.010265 \\
\hline RPS9 & 208 & 2.82127 & 0.017896 & 0.35445 & 0.009337 & 0.00958 \\
\hline KPNA2 & 205 & 2.867641 & 0.022731 & 0.348719 & 0.00263 & 0.009771 \\
\hline IL7R & 199 & 2.931496 & 0.015151 & 0.341123 & 0.002944 & 0.011601 \\
\hline LGR4 & 198 & 3.039287 & 0.020276 & 0.329025 & 3.59E-04 & 0.010662 \\
\hline CASP8 & 196 & 2.906707 & 0.020462 & 0.344032 & 0.008791 & 0.010607 \\
\hline CD247 & 196 & 2.964697 & 0.024808 & 0.337303 & 0.00539 & 0.010402 \\
\hline HIF1A & 174 & 2.950089 & 0.016343 & 0.338973 & 0.003123 & 0.013813 \\
\hline MYO19 & 173 & 2.93548 & 0.0148 & 0.34066 & 0.010687 & 0.00917 \\
\hline MNDA & 168 & 3.236498 & 0.007305 & 0.308976 & $7.84 E-04$ & 0.012766 \\
\hline
\end{tabular}

pathways, for instance, chemokine and cytokine-related pathways (i.e., TNF, IL-17, chemokine, and cytokinecytokine receptor interaction), $\mathrm{T}$ cell and B cell-related pathways (i.e., B cell receptor and Th1 and Th2 cell differentiation), NF-kappa B, and microbial infection-related pathways (i.e., Epstein-Barr virus infection and Staphylococcus aureus infection).

3.4. Identification of Hub Genes by Constructing the PPI Network. The PPI network of DEGs expressed in pulpitis shown in Figure 2 consisted of 9,070 gene nodes and 24,903 PPI interaction pairs. File S1 shows the topological characteristics of all DEG nodes in Figure 2. Table 3 shows the top 20 gene nodes were ranked in descending order according to their degree. It can be seen from Figure 4 that the gene UBD with the highest degree was identified to play the most important role in the network by interacting with the highest number of DEGs. Apart from the gene UBD, some other upregulated DEGs (e.g., IFI16, ARRB2, HLA-B, EZH2, ADRB2, LYN, FOS, RPS9, KPNA2, IL7R, CASP8, CD247, HIF1A, MYO19, and MNDA) and downregulated DEGs (e.g., SFN, MAP3K1, and LGR4) were also identified to play critical roles in the network.

3.5. Enriched Biological Processes of DElncRNAs. As shown in Figure 5, DElncRNAs were found to be significantly involved in many biological processes, for example, immune cells (dendritic cells, leukocytes, and T cells)-related BPs (e.g., regulation of dendritic cell differentiation, dendritic cell differ- entiation, $\mathrm{T}$ cell activation involved in immune response, regulation of $\mathrm{T}$ cell activation, and leukocyte differentiation), cytokine-related BPs (i.e., interferon-gamma production, regulation of cytokine secretion, and negative regulation of cytokine secretion).

3.6. The Similarity of Functional Terms of DElncRNAs. As shown in Figure 6, immune cells (e.g., lymphocytes, leukocytes, and $\mathrm{T}$ cells)-related $\mathrm{GO}$ functional terms were observed to interact with cytokine-related GO terms (e.g., the cellular response of cytokine stimulus, positive regulation of cytokine production, and regulation of interleukin-1 production).

3.7. The DElncRNA-DEG Regulatory Network. As shown in Figure 7, the DElncRNA-DEG regulatory network consisted of 312 nodes and 905 edges. File S2 shows the characteristics of all nodes in the network in Figure 7. As seen from Table 4, many lncRNAs with the highest degree play critical roles in the network, such as RP11-702F3.3, RP5-963E22.4, RP11555G19.1, CTD-2568A17.1, and PRSS29P.

3.8. Identification of Hub Transcription Factor. The TF-DEG regulatory network consisted of 1,750 nodes and 17,095 edges (Figure 8). File S3 shows the topological characteristics of all nodes in the network in Figure 8. Combining the data shown in Figure 8 and Table 5, it can be found that only one TF-FOS was differentially expressed in pulpitis among the top 20 nodes of the TF-DEG network. Although the other 19 nodes (i.e., GATA2, ETS1,YBX1, AR, FOXP3, GATA1, 


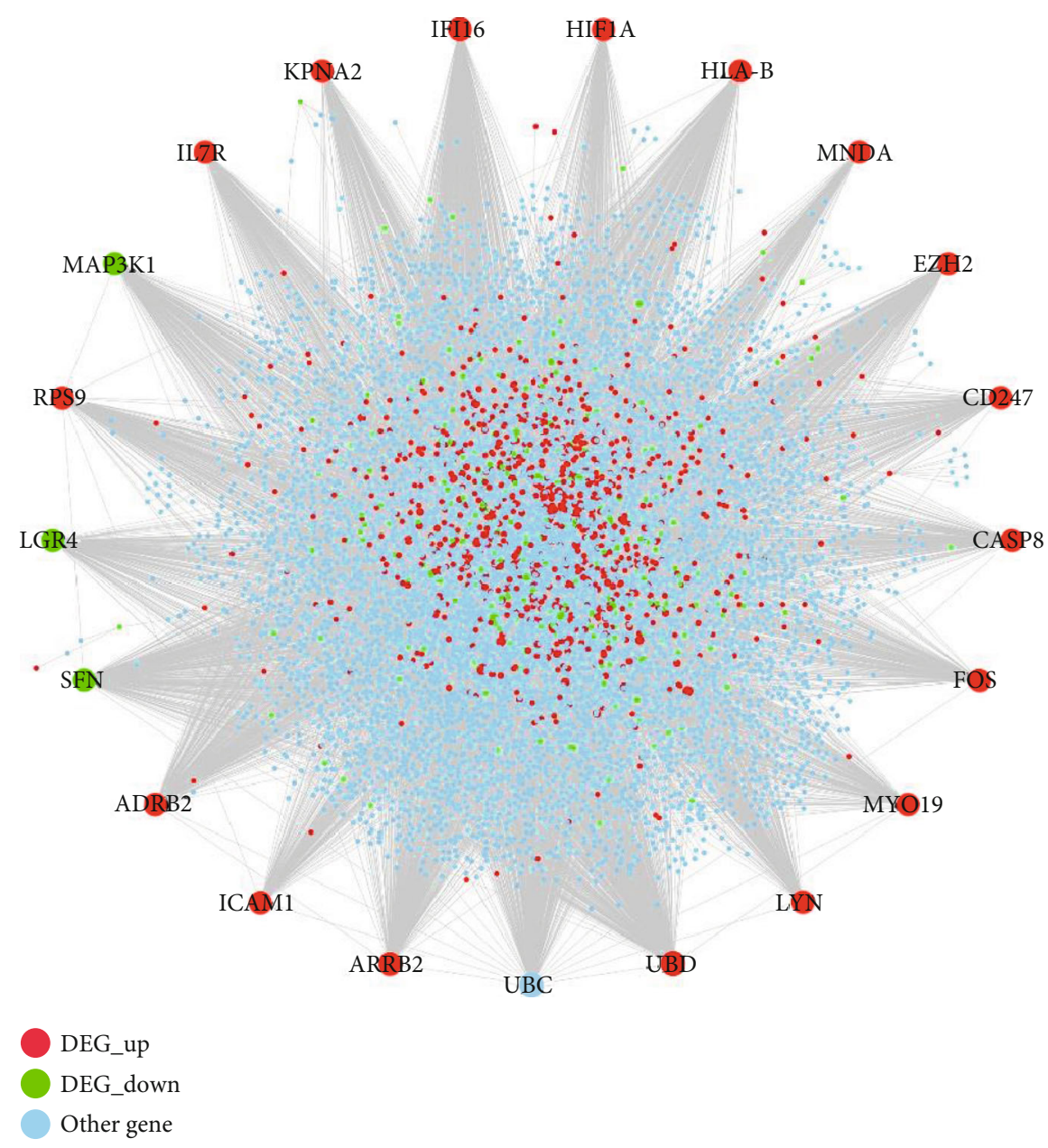

FIGURe 4: The PPI network of DEGs. The red and green circle nodes represent up- and downregulated DEGs, respectively. The sky-blue circle nodes represent the non-DEGs which interact with DEGs in the PPI network.

SP1, E2F4, PRDM14, ARNT, MIA3, JUN, CREB1, FOS, STAT1, CEBPA, AHR, E2F1, PAX5, and Pax-5) except FOS were not differentially expressed in pulpitis, they still play critical roles in the pathogenesis of pulpitis by interacting and regulating DEGs that are expressed in pulpitis. As seen from Figure 8, some other TFs were also found to be differentially expressed in pulpitis, for example, some FOSB, JUNB, EGR1, HIF1A, PLAU, MECOM, TP63, and BDNF.

3.9. Identification of Significant Gene Modules. As seen from Figure 9, five gene modules with varying colors (i.e., blue, brown, grey, turquoise, and yellow) were identified from GSE77459. Among these five coexpressed gene modules, the blue module with the lowest $p$ value was found to be the most significant module. Regarding GSE92681, eight gene modules with various colors (i.e., black, blue, brown, green, grey, red, turquoise, and yellow) were identified, among which the green module with the lowest $p$ value was found to be the most significant module.

3.10. Construction of PPI Network for Selected Significant Gene Modules. The PPI networks were constructed for these two selected significant coexpressed gene modules (blue module in GSE77459 (Figure 10) and green module in GSE92681 (Figure 11)), respectively. The PPI network of the blue module within the GSE77459 dataset consisted of 3,599 gene nodes and 4,712 edges, while the PPI network of the green module within the GSE92681 dataset consisted of 930 gene nodes and 1,019 edges. Files S4 and S5 show the topological characteristics of all nodes of the network shown in Figures 10 and 11 , respectively. Tables 6 and 7 show the topological characteristics of these two PPI networks depicted in Figures 10 and 11, respectively. Among the top 25 genes in the PPI network of the blue module of the GSE77459 dataset, only 3 upregulated DEGs (BIRC3, ITPR3, and PTPRB) were found; by contrast, the other 22 genes within the top 25 gene nodes were not DEGs (Table 6). Among the top 25 genes in the PPI network of the green module of the GSE92681 dataset, only one upregulated DEG (MMP-7) and one downregulated DEG (IK) were found; by contrast, the other 23 genes were not DEGs (Table 7).

3.11. The ceRNA Network. As shown in Figure 12, a ceRNA network consisting of DElncRNA-miRNA-DEmRNA 


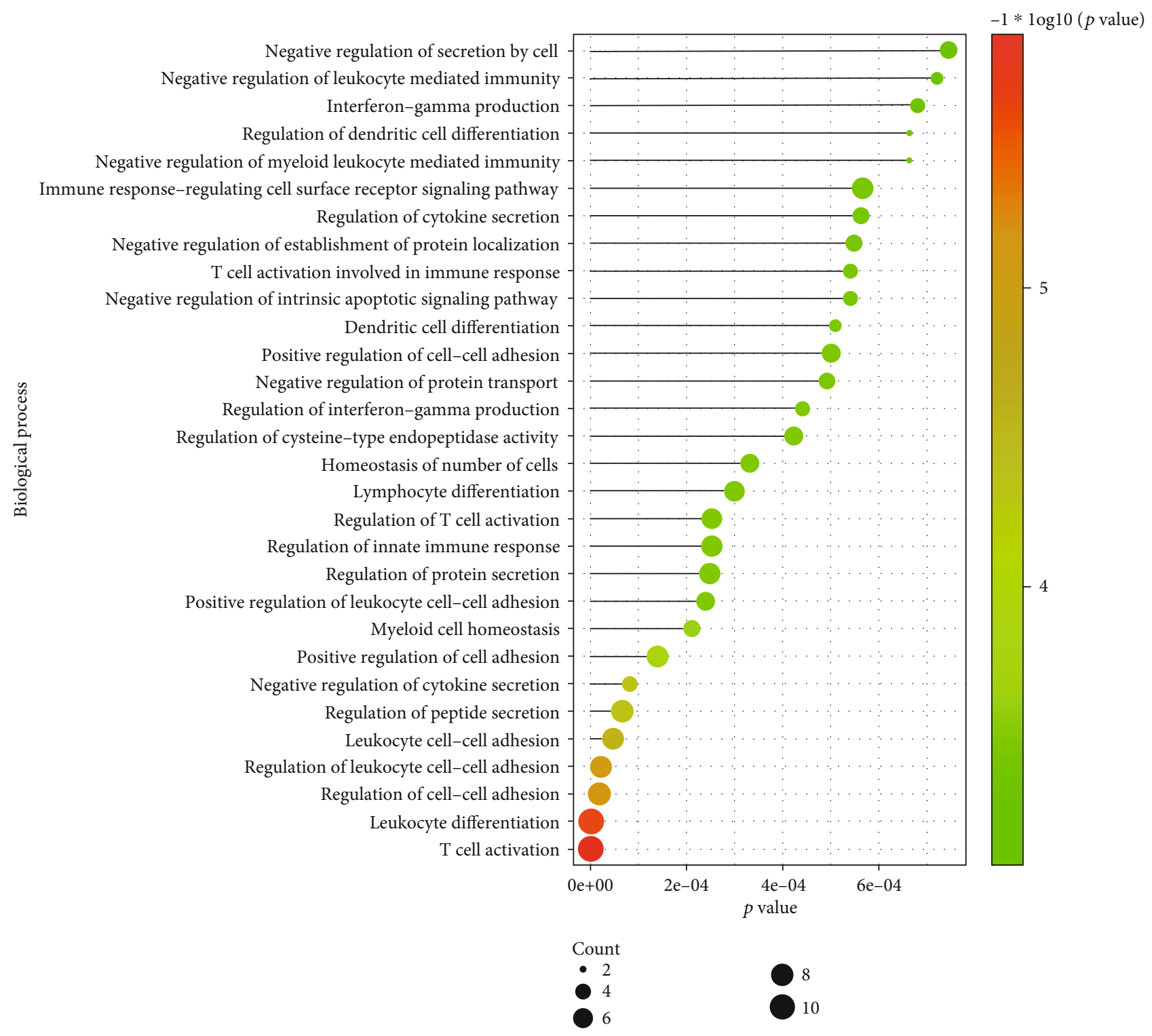

FIGURE 5: The significantly enriched biological processes of DElncRNAs. Count represents the number of genes enriched in a BP term, and $-\log 10[p$ value] represents the enrichment score. The bigger size of the dots corresponding to a BP term means more genes were enriched in this term. The colored dots represent the term enrichment: green indicates low enrichment, and red indicates high enrichment.

interaction pairs was depicted. File S6 shows the topological characteristics of all nodes in the network in Figure 12. Combined with the information of the top 20 nodes shown in Table 8, it can be observed that lncRNA XIST plays the most important role in this network. Apart from lncRNA XIST, some genes (e.g., MIR155HG, LCP1, EZH2, and NR4A1) and several miRNAs (e.g., hsa-miR-340-5p, hsa-miR-47315p, hsa-miR-5590-3p, hsa-miR-27a-3p, hsa-miR-27b-3p, hsa-miR-329-3p, hsa-miR-362-3p, hsa-miR-494-3p, hsamiR-424-5p, hsa-miR-2682-5p, hsa-miR-515-5p, hsa-miR766-5p, hsa-miR-449c-5p, hsa-miR-34a-5p, and hsa-miR449a) also play critical roles in the network. As shown in Figures 13(a)-13(c), three clusters were identified from the ceRNA network. As seen from Figure 13(c), LINC00630 can compete with two miRNAs (miR-539-3p and miR-485$3 p$ ) in targeting gene PEX5, and LINC00630 can indirectly target gene PEX5.

\section{Discussion}

This study identified many genetic and epigenetic biomarkers involved in the pathology of pulpitis, including six hub genes in the PPI network (i.e., UBD, MAP3K1, HIF1A, CASP8, IFI16, and FOS), several factors involved in the ceRNA network (e.g., three genes (i.e., LCP1, EZH2, and NR4A1), five miRNAs (i.e., miR-340-5p, miR-4731-5p, miR-27a-3p, miR-34a-5p, and miR-766-5p), and three lncRNAs (i.e., XIST, MIR155HG, and LINC00630)), six 


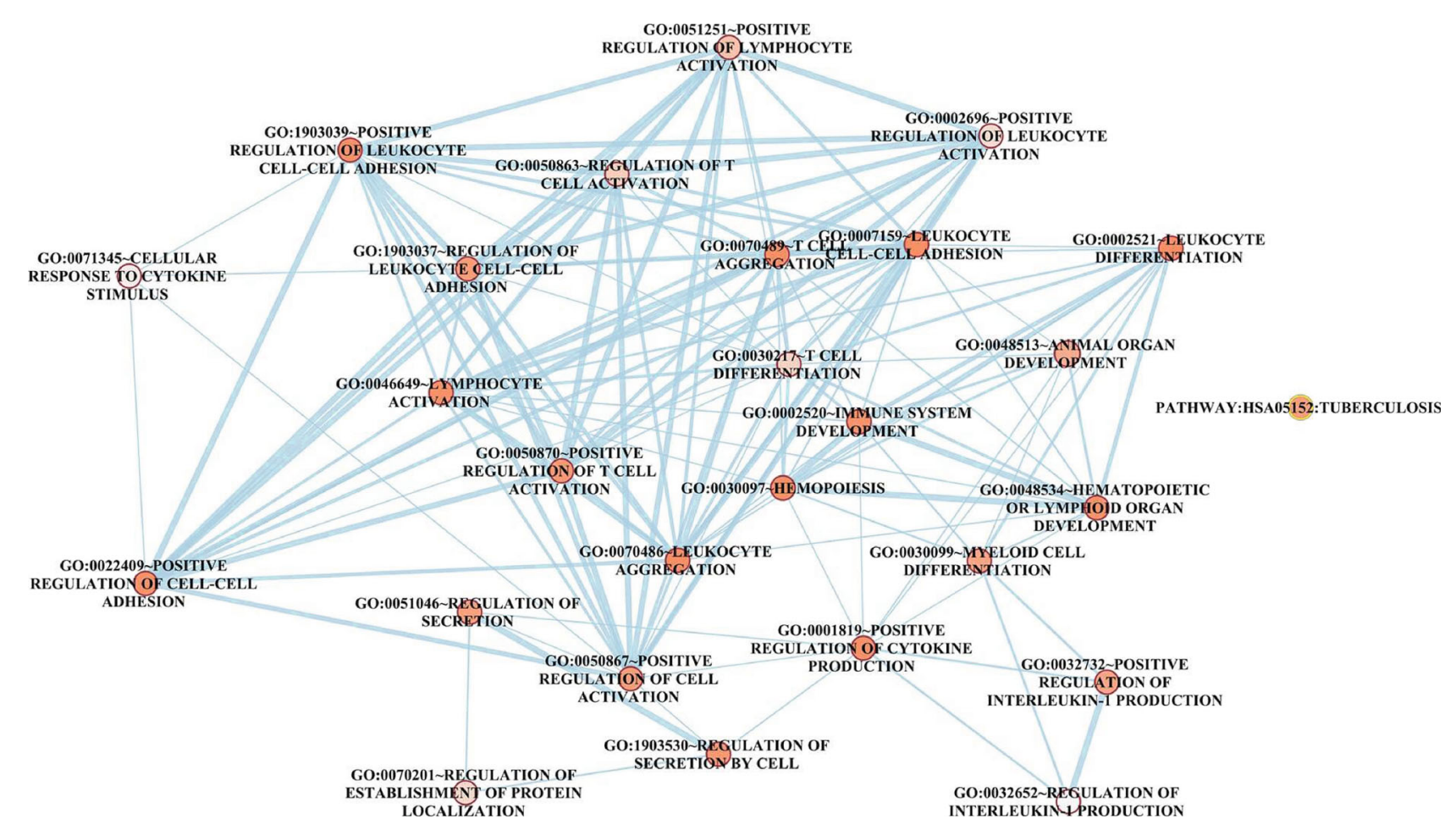

Figure 6: The enrichment map shows the GO interacting network of genes targeted by DElncRNAs. The orange circle node represents the significantly enriched GO terms, and the line represents the interaction between GO terms.

transcription factors (i.e., GATA2, ETS1, FOXP3, STAT1, FOS, and JUN), and seven signaling pathways (i.e., NODlike receptor (NLR), Toll-like receptor (TLR), NF-kappa B, tumor necrosis factor (TNF), cell adhesion molecules (CAMs), chemokine, and cytokine-cytokine receptor interaction pathway). The detailed roles of these critical factors are supported by the previous scholar evidence and will be described in the following section.

Many genes are identified to be involved in the PPI network of pulpitis; however, there is still no direct evidence that can support the involvement of these genes in pulpitis. Herein, only six genes that were most investigated by previous research were described, including UBD, MAP3K1, HIF1A, CASP8, IFI16, and FOS. For the first example, UBD (Ubiquitin D) was shown to have multiple cellular processes that occurred in pulpitis: regulating NF-kappa B signaling pathway [27], mediating cell apoptosis in a caspasedependent manner [28], and being involved in the maturation of dendritic cells [29]. Looking at the case of MAP3K1 (Mitogen-Activated Protein Kinase Kinase Kinase 1), this gene encodes a serine/threonine kinase and has been shown to be part of many signaling transduction cascades including ERK (extracellular signal-regulated kinases) [30] and JNK (cJun N-terminal kinase) kinase [31], NF-kappa B [32], TLR4 signaling [33], and IL-1 family signaling pathways [32]. Since these pathways mentioned here have been verified to be implicated in pulpitis [34-36], MAP3K1 can be speculated to be also involved in pulpal inflammation. Taking the case of HIF1A (hypoxia-inducible factor 1 subunit alpha), this gene encodes the alpha subunit of transcription factor hypoxia-inducible factor-1 (HIF-1) [37]. This gene has been shown to regulate the cellular and systemic homeostatic response to the hypoxia environment by activating many genes related to angiogenesis and apoptosis [38]. Since the hypoxic environment caused by a collapse of the venous microcirculation during the pulpal inflammation could result in localized or generalized pulp necrosis and death [39], HIF1A could be a specific signal which indicates the potential deterioration risk from irreversible pulpitis to pulp necrosis and death. For example, caspase- 8 and caspase- 9 (encoded by CASP8 and CASP9) are cysteine proteases that play a crucial role in the signaling pathways of apoptosis, necrosis, and inflammation [40]. Since CASP9 is involved in cell apoptosis in human dental pulp stem cells from deciduous teeth [41] and also activation of caspase- 9 can lead to activation of downstream caspase-8 [42], CASP8 can be therefore assumed to be involved in the signaling pathway of apoptosis in the pathogenesis of pulpitis. Another example is interferon gamma inducible protein 16 (IFI16) that is induced by IFN- $\gamma$, a member of the HIN-200 family of cytokines. A high prevalence of IFN- $\gamma$ messenger RNA in inflamed pulps has been detected [43], and the methylated status of IFN- $\gamma$ has been altered from total methylation in healthy pulp to partial methylation or unmethylation in the inflamed pulp. Since IFN- $\gamma$ cytokine may be implicated in the immune response during the process of pulp inflammation [44], the epigenetic events of pulpitis could also be relevant to the alteration of IFI16. Looking at another example, the Fos gene family (FOS, FOSB, FOSL1, and FOSL2) has been suggested to regulate the process of cell proliferation, differentiation, 


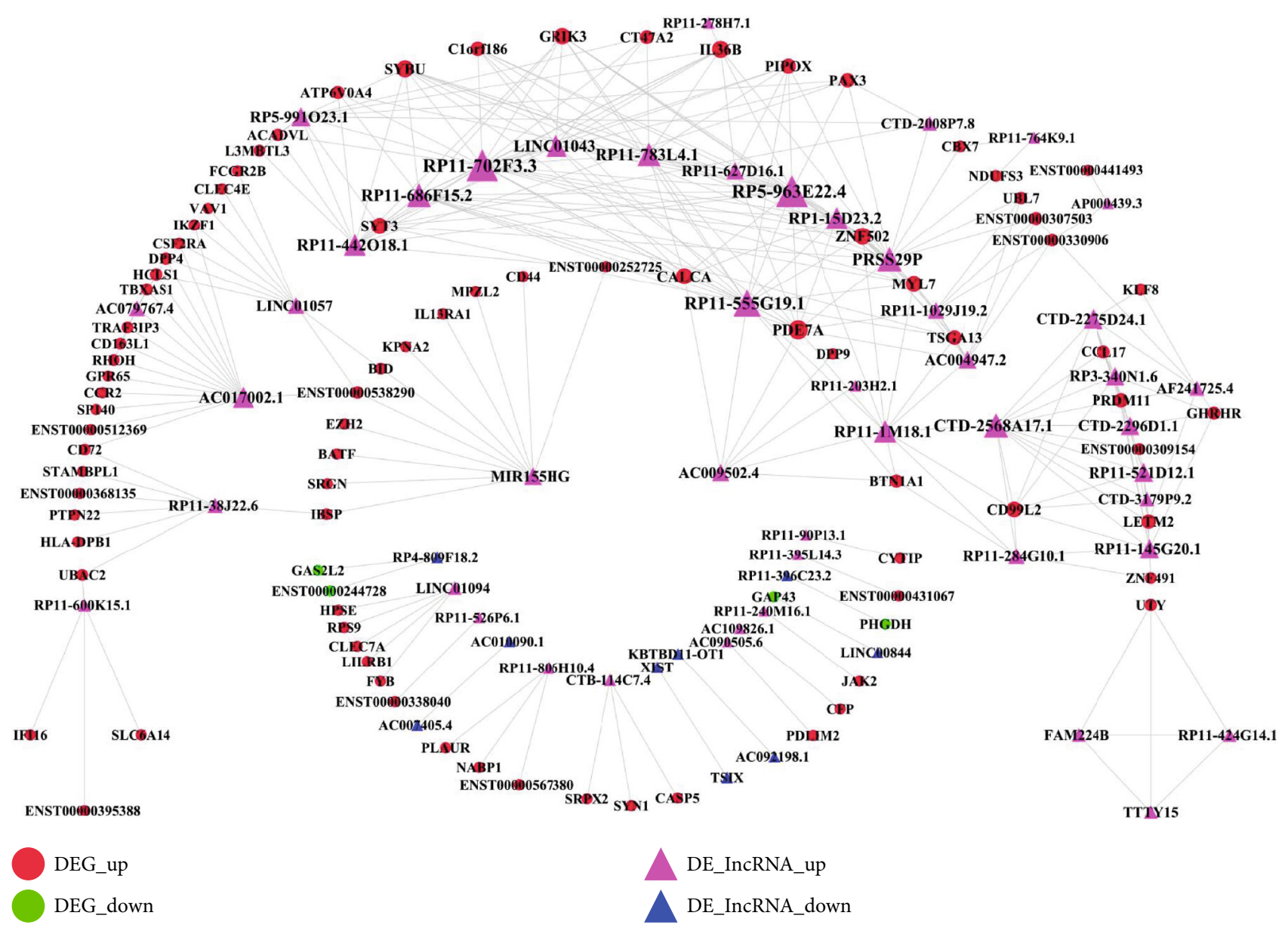

FIGURE 7: The DElncRNA-DEG regulatory network involved in pulpitis. The red circle nodes represent the upregulated DEGs, and the green circle nodes represent the downregulated DEGs. The rose-red triangle nodes represent the upregulated DElncRNA, and the blue triangle node represents the downregulated DElncRNA.

transformation, and apoptosis. The expression of the immediate-early gene product Fos was reported to be evoked by the LPS-induced pulpal inflammation in the rostral trigeminal regions of ferrets [45]. Regarding the pattern of its expression, another study using the rat model found that the expression of Fos induced by chronic tooth pulpal inflammation in dynorphin-rich regions of rat brainstem was shown to be temporal and spatial [46]. The role of almost all of the genes in pulpitis is based on speculation and thus needs to be validated in future research by designing relevant experiments.

Three genes (i.e., LCP1, EZH2, and NR4A1) are identified to be key biomarkers in the ceRNA network. For example, LCP1 (Lymphocyte Cytosolic Protein 1) is significantly enriched in a GO term named T cell activation [47]. The activation of $\mathrm{T}$ lymphocytes can orchestrate other types of immunocompetent cells, thereby promoting the local immune defense that occurred in the dental pulp [48]. Given this evidence, it can be assumed that LCP1 might be involved in the pathogenic mechanism of pulpitis by regulating $\mathrm{T}$ cellmediated immune response. For another example, the enhancer of zeste homolog 2 (EZH2), as a catalytic subunit of PRC2 (polycomb repressor complex 2), could regulate gene silencing via its histone methyltransferase activity, accumulation of DNA damage, and chromosome abnormalities [49]. EZH2 is suggested to be implicated in the pulp inflammation, proliferation, and regeneration by inhibiting osteogenic differentiation of human dental pulp cell (HDPCs) and enhancing inflammatory response and proliferation [50]. Another research investigating the effect of EZH2 in odontogenic differentiation of hDPCs suggested that EZH2 could impair mineralization of HDPCs under the mechanism of activating the Wnt canonical signaling pathway [51]. Taking the final example, Nuclear Receptor Subfamily 4 Group A Member 1 (NR4A1) is enriched in many pulpitis-related signaling pathways, including signaling by PDGF and EGFR, PI3K/AKT activation, and MAPK signaling pathways, and also some GO terms including positive regulation of endothelial cell proliferation and apoptotic process. The previous scholar evidence investigating the involvement of NR4A1 in inflammation showed that the overexpression of NR4A1 was associated with a chronic low-grade inflammatory state [52] and also plays a key role in mediating the antiinflammatory effects of apoptotic cells [53]. However, the expression patterns and its regulatory mechanisms of NR4A1 remain to be researched in pulp inflammation. 


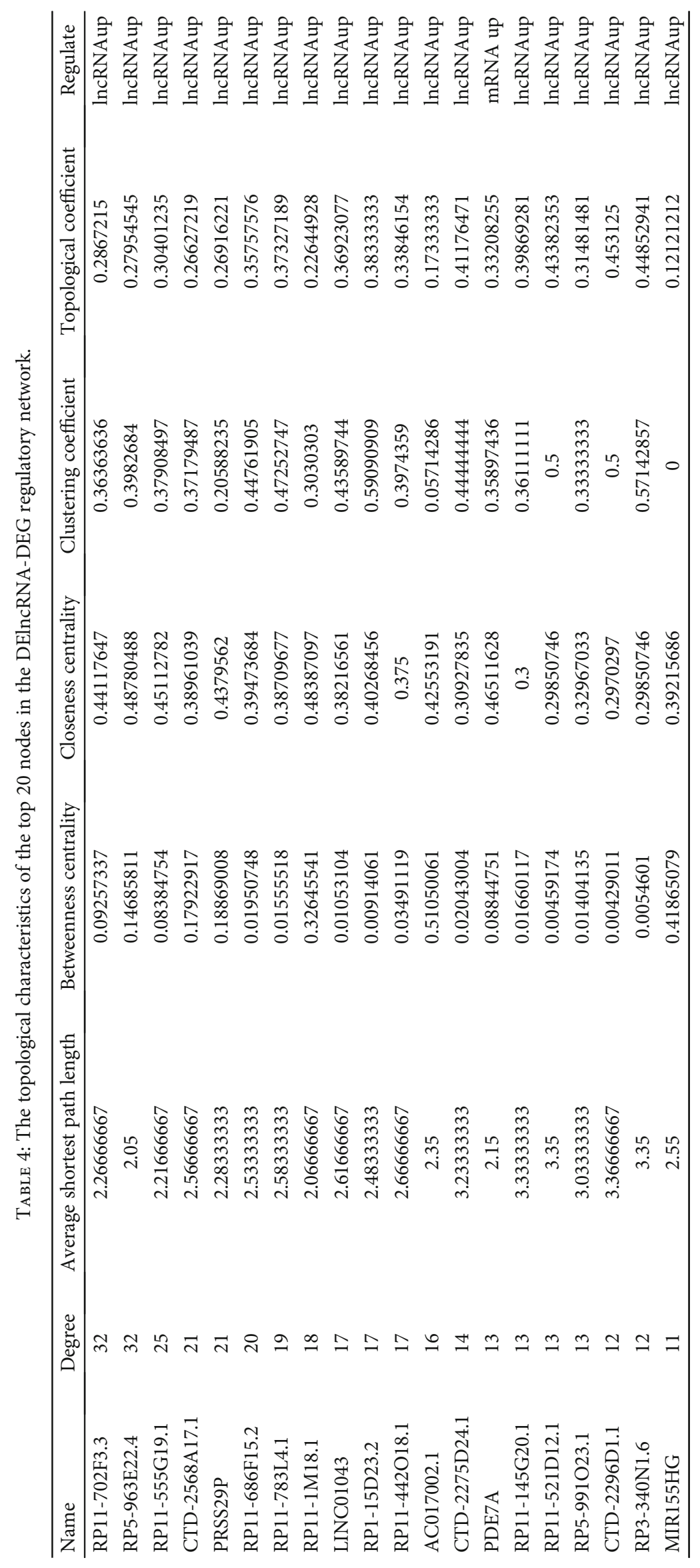




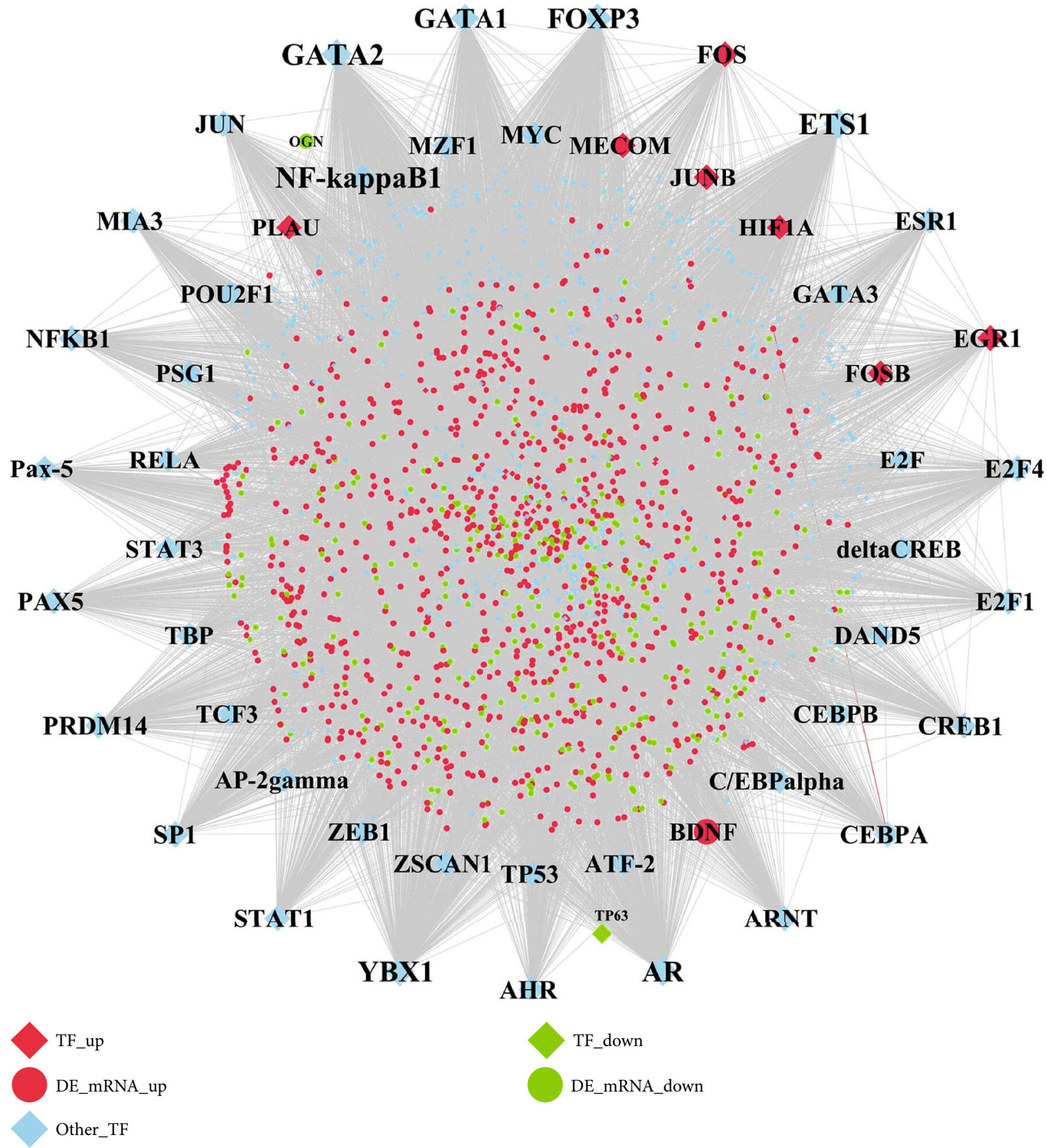

FIGURE 8: The TF-DEG regulatory network involved in pulpitis. The diamond nodes represent TFs and the circle nodes represent DEGs. For the diamond nodes, the red diamond nodes represent the upregulated TFs, the green diamond nodes represent the downregulated TFs, and the sky-blue diamond nodes represent other nondifferentially expressed TFs. For the circle nodes, red circle nodes represent the upregulated DEGs, while the green circle nodes represent the downregulated DEGs.

Many miRNAs are involved in the ceRNA network of pulpitis; however, the expression patterns and functions of almost all of them have not been investigated in pulpal inflammation. Based on the potential target genes of the miRNAs searched on the miRWalk database [54], some miRNAs (i.e., miR-340-5p, miR-4731-5p, miR-27a-3p, miR-34a-5p, and miR-766-5p) could be assumed to be implicated in pul- pitis by targeting genes related to inflammatory response and regeneration. For the first example, miR-340-5p has been validated to target gene LIMS1 (LIM Zinc Finger Domain Containing 1), the encoded protein of which is involved in the integrin signaling [55]. Sine integrin-associated signaling is implicated in the odontogenic stimulation of human dental pulp stem cells [56]; miR-340-5p might be involved in the 


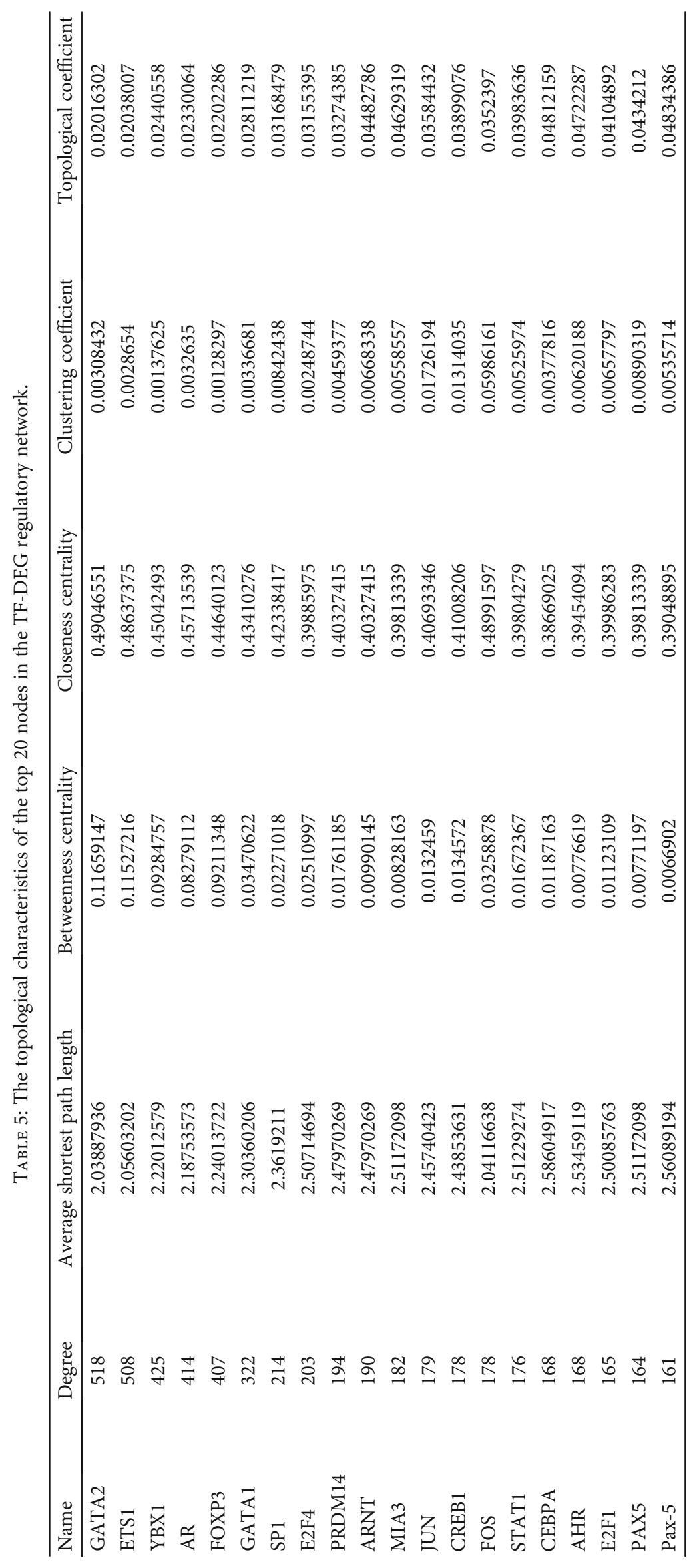


GSE77459

Module significance $p=8.3 \mathrm{e}-63$

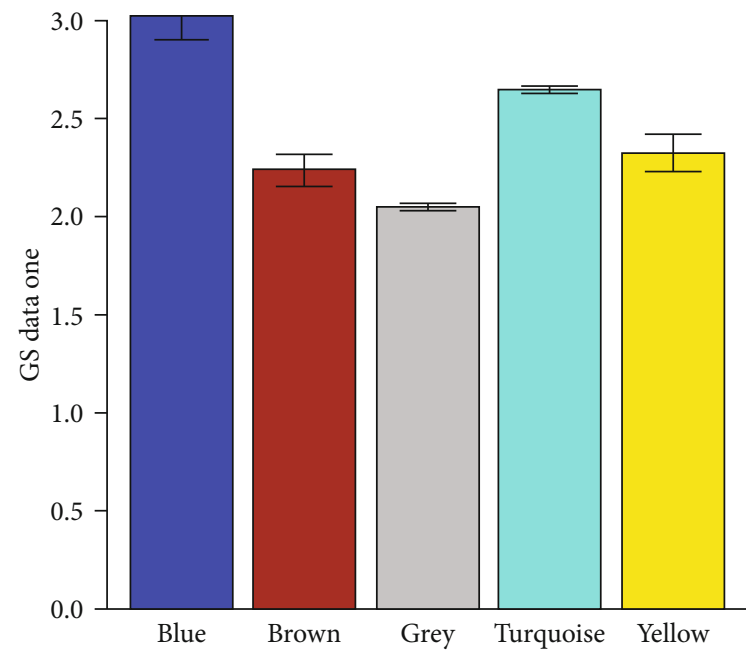

GSE92681

Module significance $p=1.6 \mathrm{e}-16$

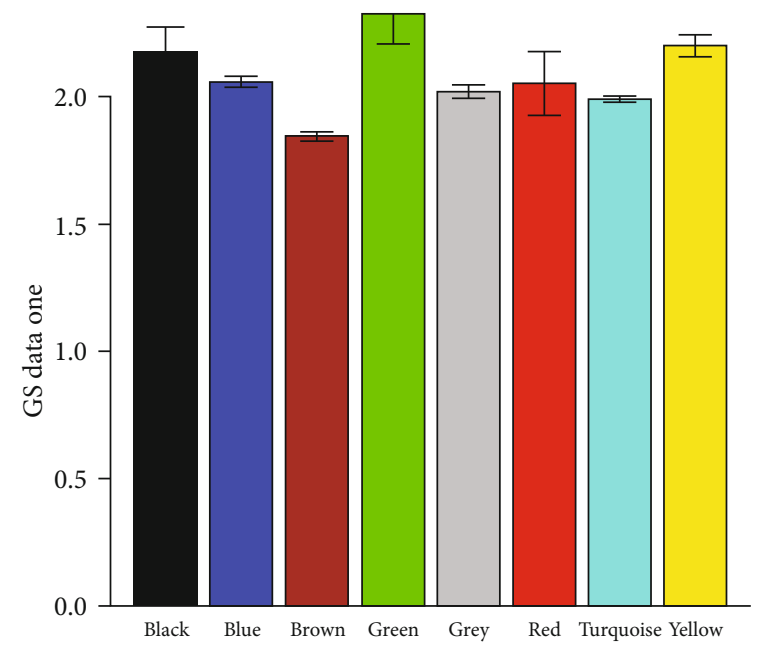

FIGURE 9: The coexpressed gene modules identified in GSE77459 and GSE92681. The horizontal axis represents each different color module; the vertical axis represents the correlation coefficient between genes in each module and disease status.

pulp healing and regeneration during the pathogenic processes of pulpitis. In the case of miR-4731-5p, it has been validated to target gene IRAK4 (Interleukin 1 ReceptorAssociated Kinase 4), which encodes a kinase that can activate the upregulation of NF-kappa B [57]. Since NF-kappa $\mathrm{B}$ has been found to be activated by lipopolysaccharide (LPS) and tumor necrosis factor (TNF) in the dental pulp stem cells (DPSCs) and further implicated in the immune response of pulpal infection [35], miR-4731-5p could be regarded as an inflammatory biomarker during the pulpal inflammation. In the case of miR-27a-3p, it has been found to regulate the cell proliferation of vascular endothelial cells positively and further being implicated in the angiogenesis and neovascularization through ERK1 and ERK2 cascade [58]. Since an increased number of blood vessels have been found in the inflamed human dental pulp [59], miR-27a-3p could be involved in the pulpal regeneration by mediating angiogenesis during the process of pulpitis. Taking the example of miR-34a-5p, it has been validated to target the gene MAP2K1 (Mitogen-Activated Protein Kinase Kinase 1), which encodes a dual-specificity kinase that has been wellknown to be involved in the ERK pathway [60]. Since the MAP/ERK pathway is implicated in the differentiation and stimulation of odontoblasts during reactionary dentinogenesis [61], miR-34a-5p might be involved in the dentinogenesis-based repair mechanism during the pathogenesis of pulpitis. In the case of miR-766-5p, it is one of the subtypes of miR-766 which is upregulated in inflamed pulpitis compared to the normal pulps [62]. miR-766 can target Heat Shock Transcription Factor 1 (HSF1), which encodes a transcription factor that can be rapidly induced after temperature stress [63]. Since thermal stresses, including hot and cold loadings, may induce the activation of tooth pain signaling [64], miR-766 could be assumed to be a sensitive biomarker of thermal exposure.
Three lncRNAs (i.e., XIST, MIR155HG, and LINC00630) are identified to be key factors involved in the ceRNA network of pulpitis. For the first example, the lncRNA X Inactive Specific Transcript (XIST), as a $17 \mathrm{~kb}$ long RNA transcribed by the inactive $\mathrm{X}$ chromosome, is involved in the $\mathrm{X}$ chromosome inactivation in female mammals, thus providing dosage equivalence between males and females [65]. More and more scholarly evidence has shown that XIST is dysregulated in many cancers and inflammatory conditions [66-69]. A recent study found that XIST can mediate the inflammation process of mammary epithelial cells by regulating the NF$\kappa \mathrm{B} / \mathrm{NLRP} 3$ inflammasome pathway [70]. In the case of the lncRNA MIR155HG (MIR155 Host Gene), it is formerly known as BIC (B-cell integration cluster) and has been shown to function as a primary micro (mi)RNA for miR155 [71]. Since miR-155 has been established to be an ancient master regulator of the immune response [72], the MIR155HG/miR-155 axis may be involved in many physiological and pathological processes including inflammation and immunity [73]. In the case of LINC00630, this lncRNA can interact with miR-539-3p, miR-485-3p, and PEX5 gene and combinedly generate a closed regulatory loop in the ceRNA network. The gene PEX5 (Peroxisomal Biogenesis Factor 5) encodes the type 1 peroxisomal targeting signal (PTS1) receptor, which is one of 15 peroxins required for peroxisome biogenesis [74]. A recent study showed that peroxisomes could resolve microbial infection by modulating many innate immune-related pathways (reactive oxygen species (ROS) and reactive nitrogen species (RNS) signaling) and activating the stress response kinase p38 [75]. Based on the finding of the PEX5/LINC00630/miR-539-3p/miR-485$3 \mathrm{p}$ loop, this loop may be required for promoting the immune response in pulpal inflammation.

Several transcription factors have been identified to be involved in the TF-gene regulatory network of pulpitis, 


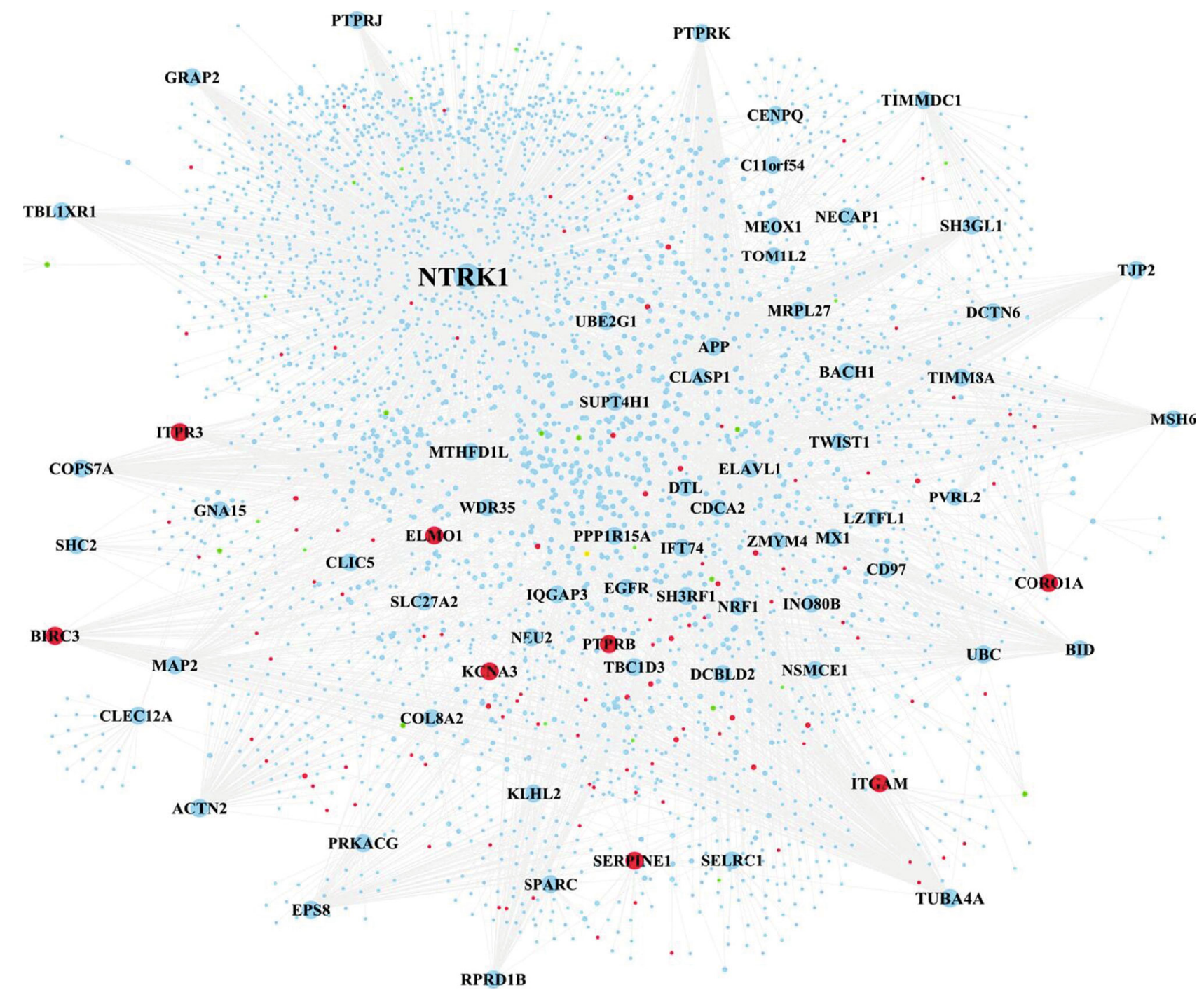

FIGURE 10: The PPI network of significant modules within the blue module in GSE77459. The red and green circle nodes represent up- and downregulated DEGs, respectively. The sky-blue circle nodes represent the non-DEGs which interact with DEGs in the PPI network.

including GATA2, ETS1, FOXP3, STAT1, FOS, and JUN. GATA2 (Endothelial Transcription Factor GATA-2) is a transcriptional activator that regulates the expression of the endothelin-1 gene in endothelial cells [76]. It has been shown that endothelial cells can influence DPSCs by secreting endothelin-1 and further promoting the odontogenic differentiation of DPSCs [77]; thus, GATA2 can be assumed to be involved in the restoration and regeneration of dental pulp. Taking the example of ETS1, it could be speculated to be involved in the inflammation and regeneration of pulp based on its dual functions: controlling the expression of many cytokines as well as chemokine genes [78], being implicated in angiogenesis by regulating the expression of genes that are associated with migration and invasion of endothelial cells [79]. Taking the case of FOXP3 (Forkhead Box $\mathrm{P} 3$ ), it is the most specific biomarker of regulatory $\mathrm{T}$ cells (Treg) $[80,81]$. Treg as a subset of $\mathrm{T}$ lymphocytes has been playing a pivotal role in the immune and inflammatory response of pulpitis by secreting anti-inflammatory cytokines, including interleukin-10 and transforming growth factor b (TGF-b) [82]. Based on this, FOXP3 can be speculated to be involved in the inflammatory response by regulating the cytokine genes. In the case of STAT1 (Signal Transducer And Activator Of Transcription 1), it has been shown to play a critical role in mediating the cellular responses to many inflammatory mediators involved in pulpitis, including interferons (IFNs), cytokines (IL1, IL6, and KITLG/SCF), and growth factors (epidermal growth factors (EGF) and platelet-derived growth factor (PDGF)) [83]. For example, IFN-gamma has been shown to be a feasible modulator to improve the dentinogenic and immunosuppressive functions of irreversible pulpitis-DPSCs [84]; cytokines as a crucial part of host response could be regarded as diagnostic markers of pulpal inflammation [85, 86]; and growth factors can contribute to the angiogenic response of pulp tissue and enhance the regeneration of pupal-like tissue $[87,88]$. Taking the final 


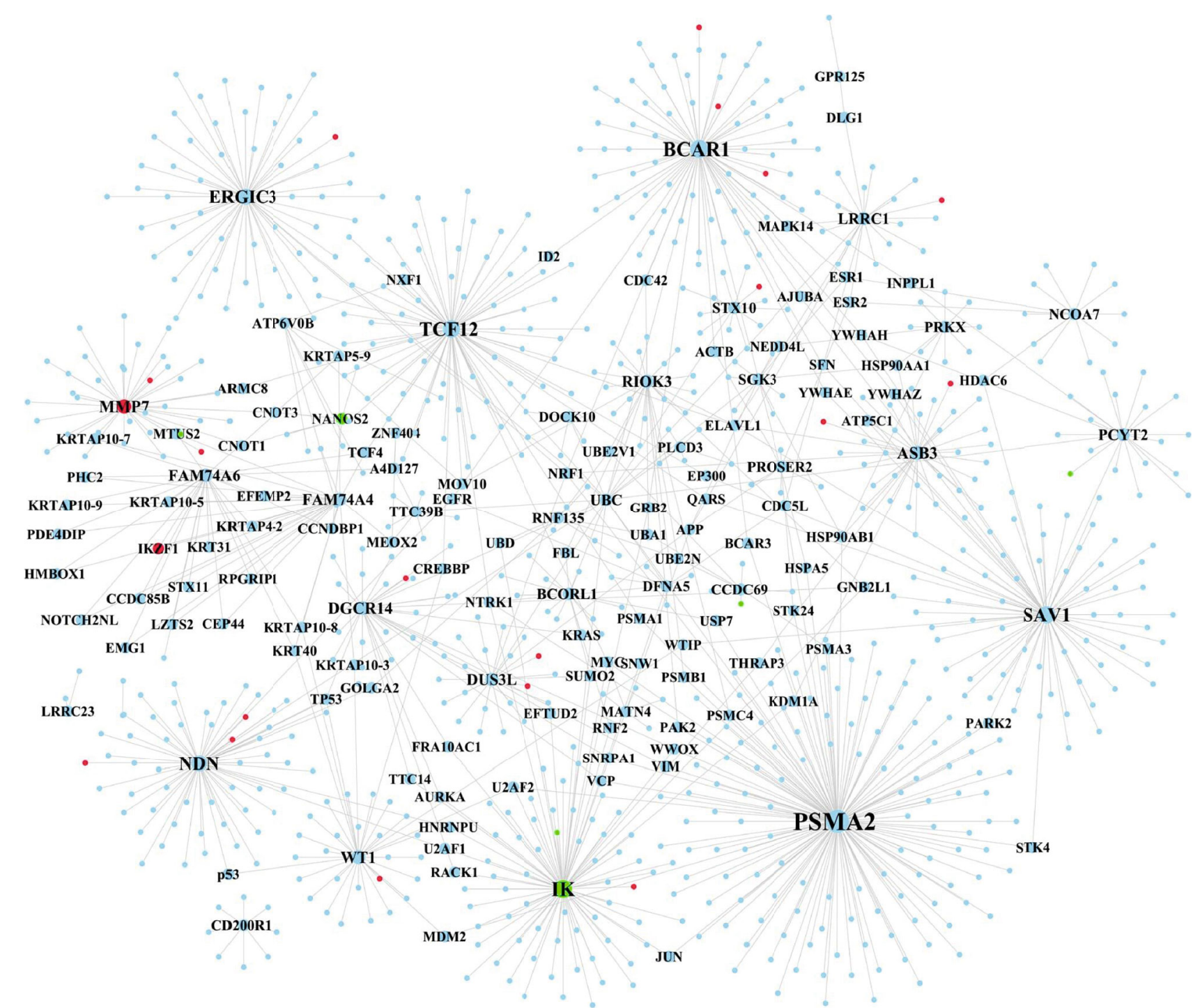

FIGURE 11: The PPI network of significant modules within the green module in GSE92681. The red and green circle nodes represent up- and downregulated DEGs, respectively. The sky-blue circle nodes represent the non-DEGs which interact with DEGs in the PPI network.

example, AP-1 complex consisting of c-JUN and c-FOS can synergize with $\mathrm{Smad} 3 / \mathrm{Smad} 4$ protein and further cooperatively mediate the transforming growth factor-beta (TGFbeta) signaling pathway [89]. Since TGF-beta has been well known to stimulate odontoblast cells to secrete reactionary dentin [90], JUN and FOS can be speculated to play a role in the repair and regeneration process of the dental pulp.

Seven signaling pathways have been identified to be significantly enriched in the pathogenesis of pulpitis, for example, NOD-like receptor (NLR), Toll-like receptor (TLR), NFkappa B, tumor necrosis factor (TNF), cell adhesion molecules (CAMs), chemokine, and cytokine-cytokine receptor interaction pathways. All of the pathways listed above have been well supported by previous studies. In the first example, the nucleotide-binding oligomerization domain- (NOD-) like receptors (NLRs) and Toll-like receptors (TLRs) are two members of the pattern recognition receptor (PRR) family. It has been shown/demonstrated by authors that PRR family members can recognize caries pathogen-associated molecular patterns (PAMPs) and play crucial roles in the initiation of dental pulp innate immunity [91]. In another example, the downregulation of the NF-kappa B gene was suggested to enhance the odontogenic differentiation of DPSCs and the formation of the collagen matrix, indicating that NF-kappa B could be a potential target for promoting pulp tissue regeneration [92]. Taking the tumor necrosis factor- $\alpha$ (TNF- $\alpha)$ as an example, it has been shown that TNF- $\alpha$ is a pleiotropic cytokine that is upregulated in pulpal tissues of teeth with irreversible pulpitis [93]. Not only does TNF$\alpha$ play a role in promoting inflammation by recruiting leukocytes and stimulating the production of proinflammatory cytokines, but it may also cause pain hypersensitivity by directly acting on nociceptive neurons [94]. In the case of cell adhesion molecules (CAMs), diverse CAM molecules (e.g., platelet-endothelial cell adhesion molecule-1 (PECAM-1), intercellular adhesion molecule-1 (ICAM-1), intercellular 


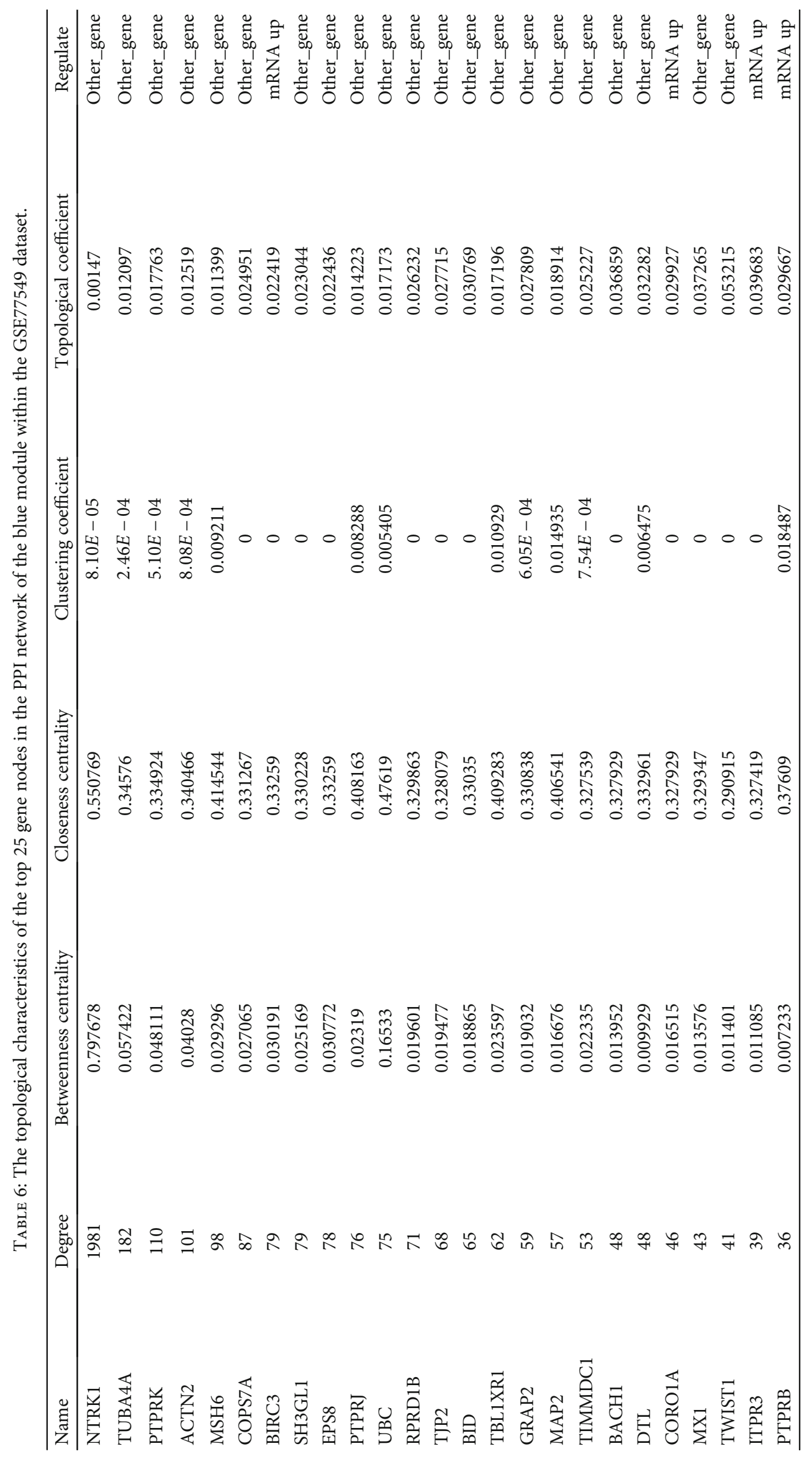




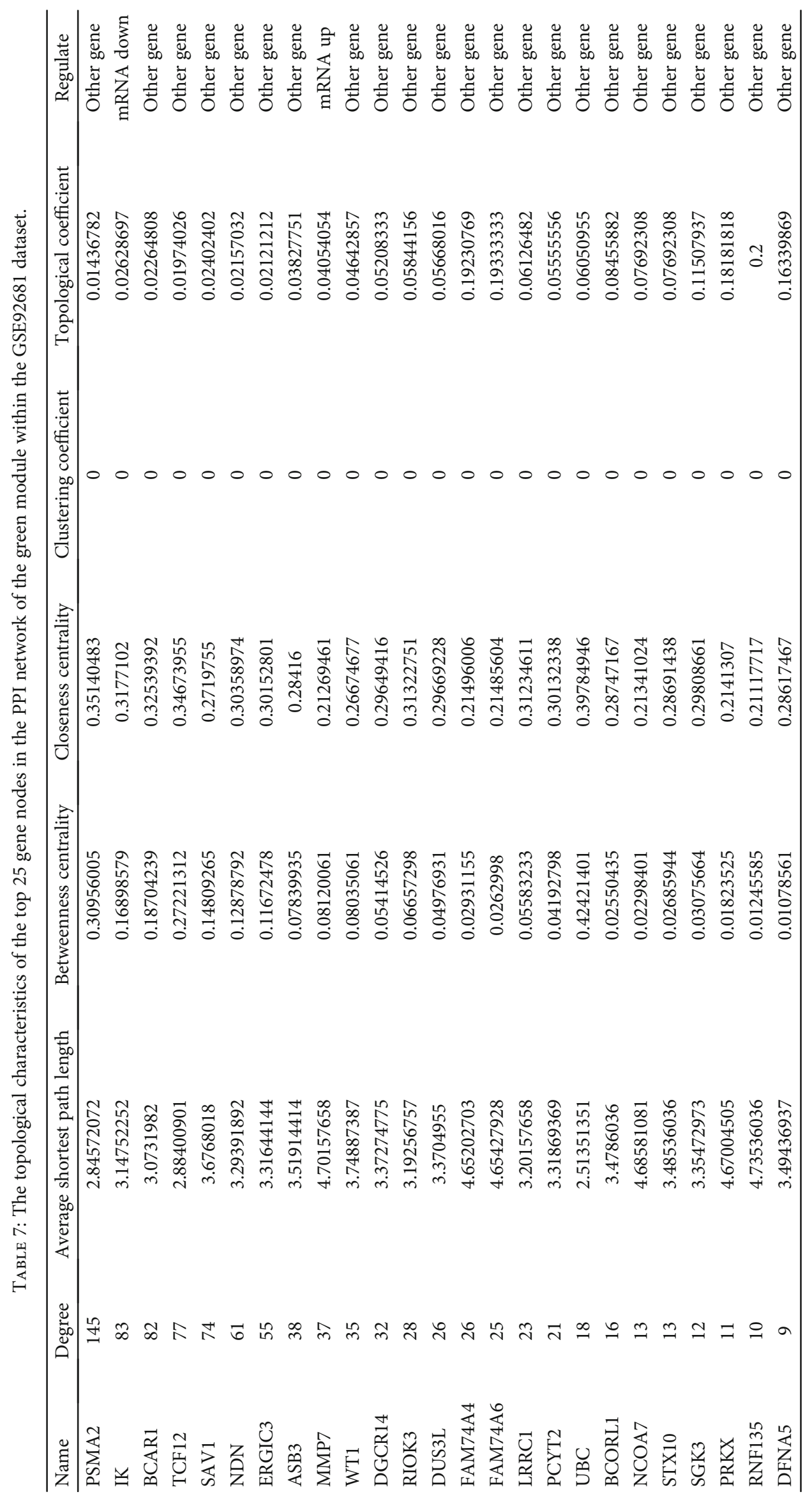




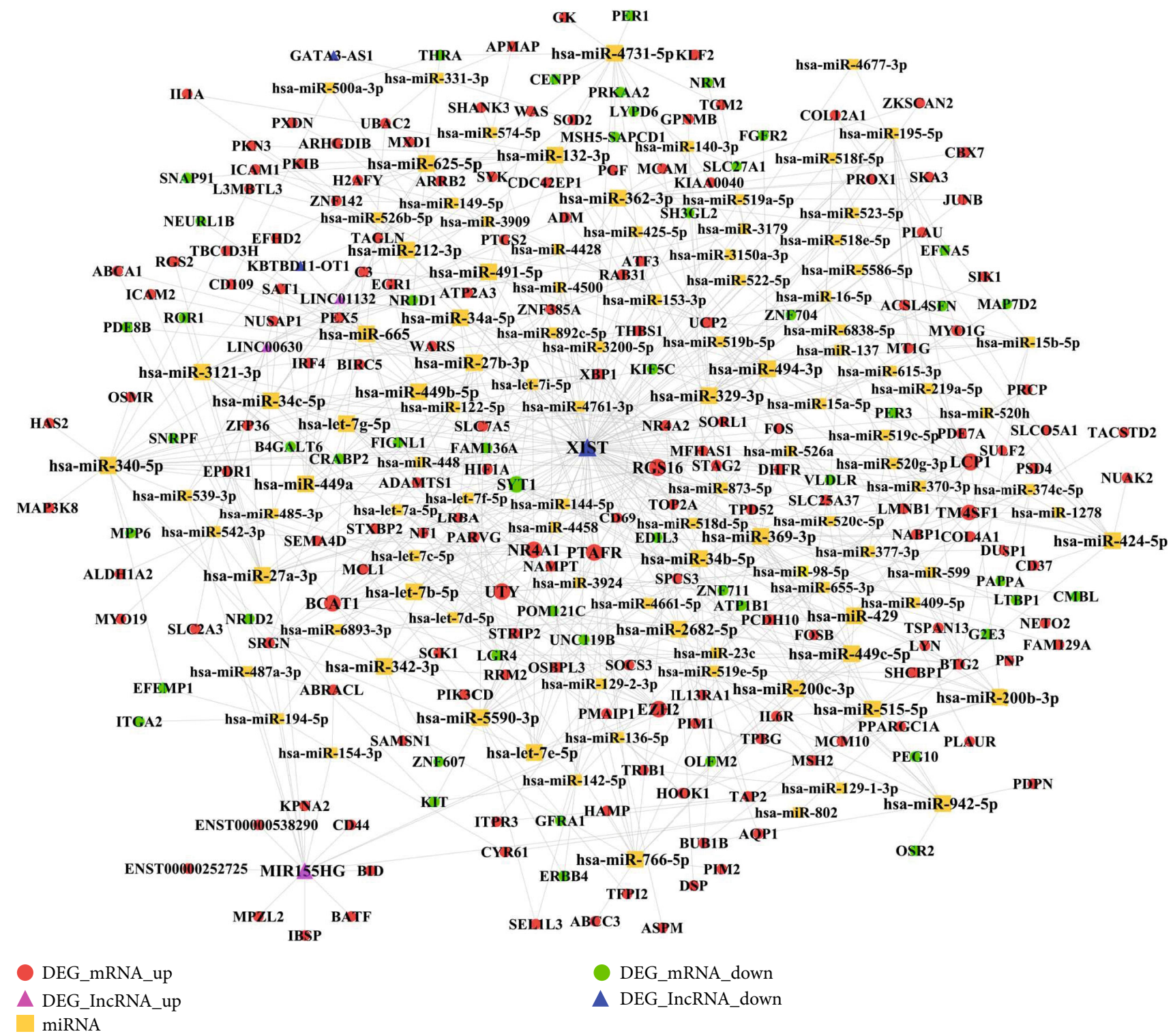

FIGURE 12: The ceRNA network consisting of DElncRNA-miRNA-DEG interaction pairs. The red circle nodes represent the upregulated DEGs, and the green circle nodes represent the downregulated DEGs. The yellow square nodes represent miRNA. The rose-red triangle nodes represent the upregulated DElncRNA, and the blue triangle node represents the downregulated DElncRNA.

adhesion molecule-3 (ICAM-3), and vascular cell adhesion molecule-1 (VCAM-1)) were shown to be expressed in the vascular endothelium of the inflamed human dental pulp, by playing roles in promoting transendothelial migration of leukocytes from the bloodstream into tissue [95]. Finally, chemokines and cytokines are kinds of inflammatory mediators suggested being involved in the innate immune response of pulpitis, playing protective roles in attracting varying inflammatory cells, inducing antibacterial reactions by the production of antimicrobial peptides such as defensins, and further killing cariogenic microbial [96]. All of the signaling pathways listed above can form complicated interactions and are involved in the inflammatory immune response of pulpitis. However, it is also worthwhile to note that the path- ways identified in this study have also been documented as the classic pathways involved in all inflammatory diseases and are not specific for pulpitis. It is therefore questionable to regard these pathways as therapeutic targets that can inhibit the progression of pulpitis.

Some limitations should be acknowledged in this study. First, only expression profiling datasets of lncRNAs and genes could be obtained, and there were no miRNA expression profile datasets related to pulpitis in the GEO dataset. It was therefore impossible to predict the expression tendency of miRNAs in the pathology of pulpitis. This also means that miRNA sequencing technology needs to be applied to investigate the alteration of miRNAs in pulpitis. Second, the sample size of the datasets included was small 


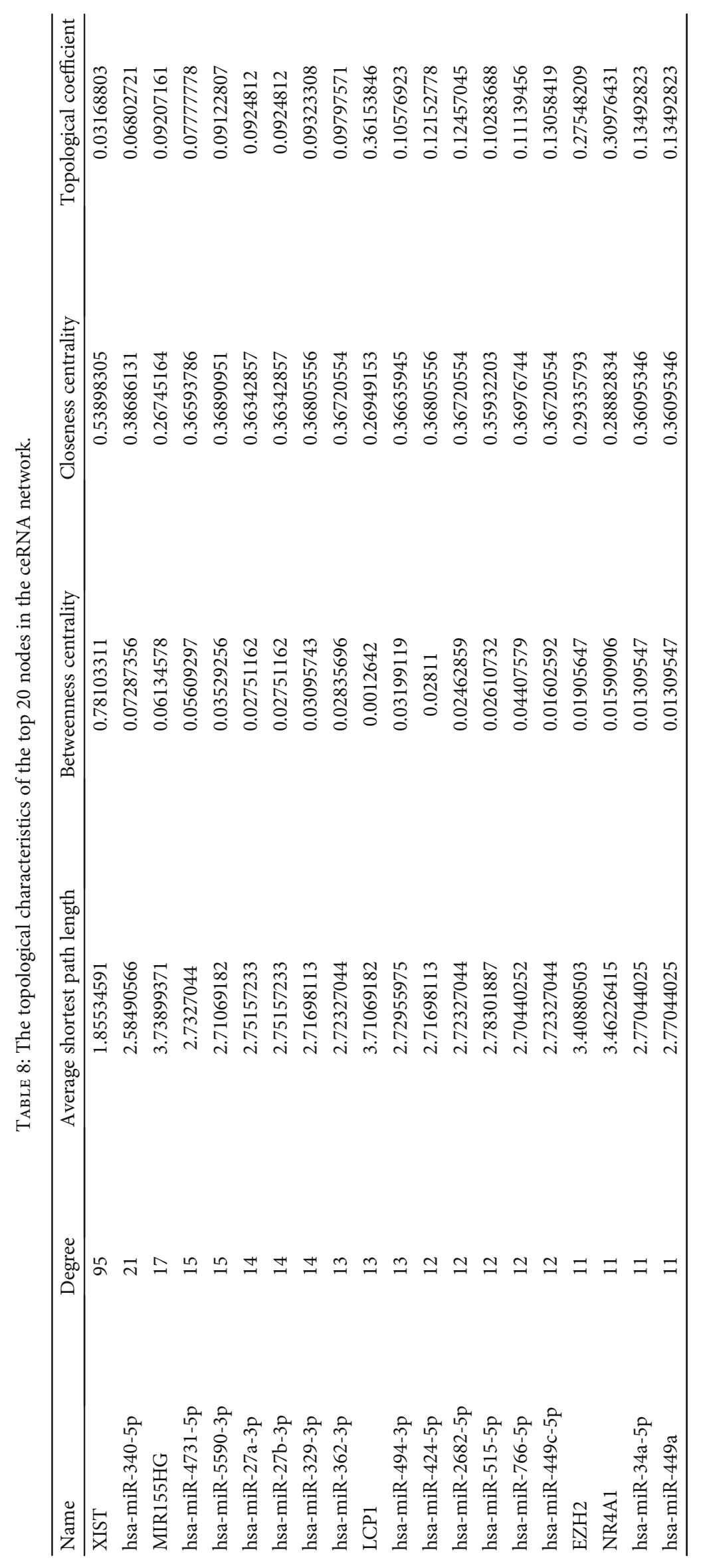




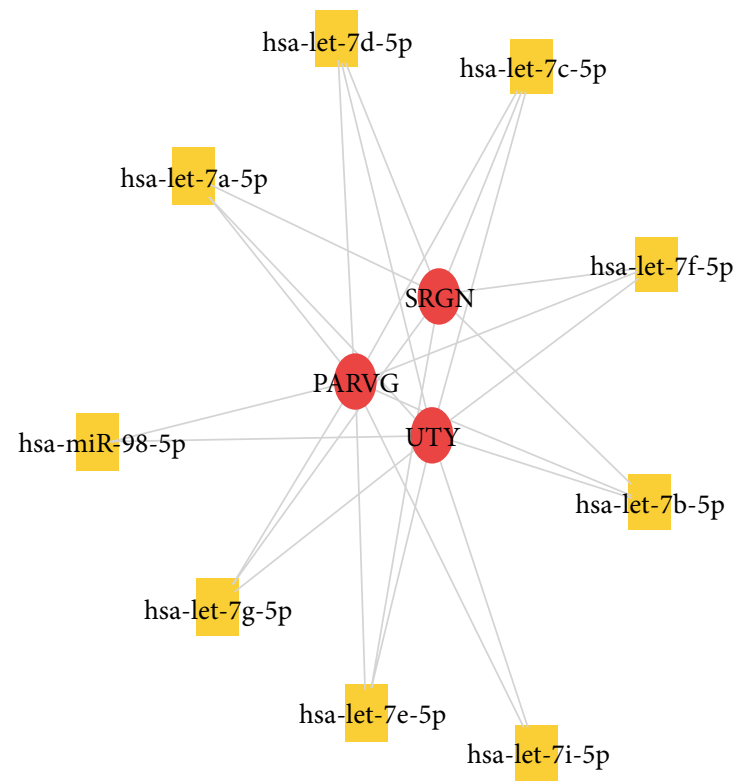

DEG_Up

miRNA

(a)

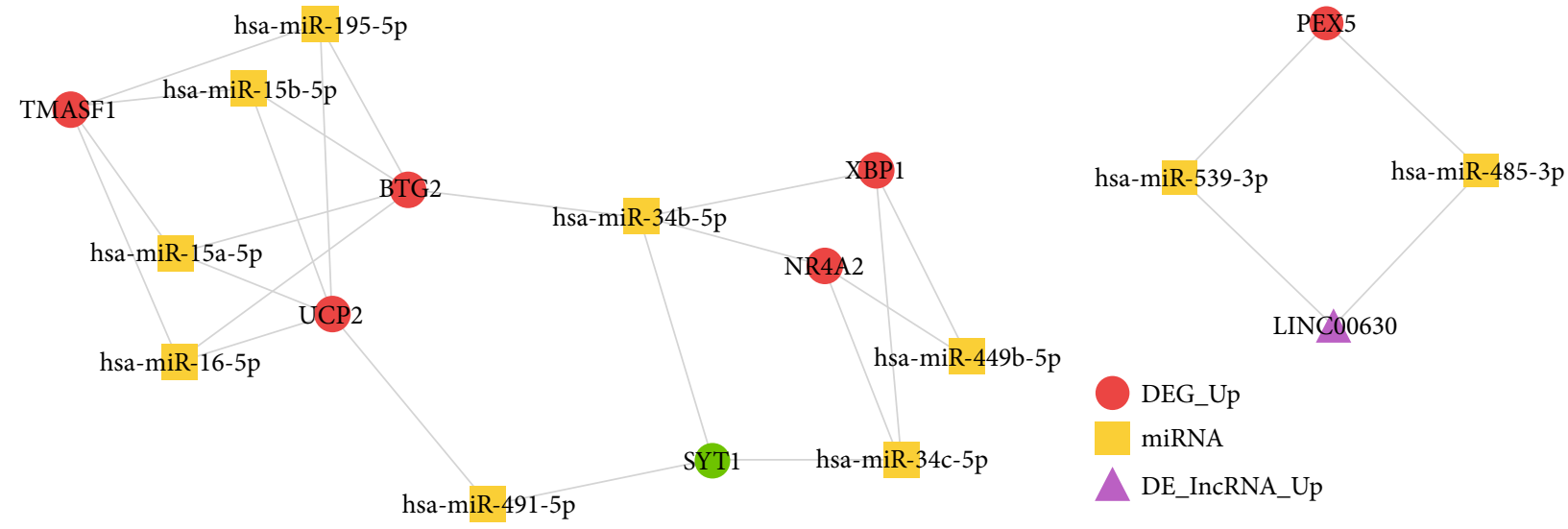

DEG_Up

miRNA

DEG_Down

(b)

(c)

FIgURE 13: Three clusters identified in the ceRNA network. The red circle nodes represent the upregulated DEGs, and the green circle nodes represent the downregulated DEGs. The yellow square nodes represent miRNA. The rose-red triangle nodes represent the upregulated DElncRNA.

(GSE92681: 12; GSE77459: 12), and the analysis based on this limited sample data may result in a decrease of the prediction accuracy. Third, it should be noted that only bioinformatic techniques were employed. And because of limited funding, no clinical experiments were performed to validate the expression of the RNA molecules predicted in pulpitis. Although this study has some limitations, the findings also provide some direction for future research. First, the biomarkers identified could be promising therapeutic targets that can lay the groundwork for future experimental research design. Second, the identification of these biomarkers can benefit the research of pulp tissue engineering, based on the evidence that genetically modified stem cells will receive better treatment efficacy compared with stem cells alone. The combined application of these genetic and epigenetic biomarkers modified DPSCs and already validated biomaterial scaffold (e.g., collagen, poly (lactic) acid, and fibrin) is promising for future regenerative endodontic therapy. 


\section{Data Availability}

The data used to support the findings of this study are available from the corresponding author upon reasonable request.

\section{Ethical Approval}

As this study only applied bioinformatic techniques based on computational analyses, all of the data from pulp tissue samples were obtained from the public datasets, and original human samples were not analyzed. Therefore, this study does not require ethical approval.

\section{Consent}

Consent for publication is not applicable in this study because no individual person's data was used.

\section{Conflicts of Interest}

The authors declare no potential conflict of interest with respect to the authorship and publication of this paper.

\section{Authors' Contributions}

Dr. Wanchen Ning (email: wanchenning0627@gmail.com) and Dr. Xiao Jiang (email: jiangxiao_524@163.com) are equally the senior authors. Dr. Hanluo Li (email: ala1004@gmail.com), Dr. Simin Li (email: simin.li.dentist@gmail.com), and Prof. Dr. Xianda Hu (email: hellocean@hotmail.com) are equally the corresponding authors.

\section{Acknowledgments}

Dr. Wanchen Ning received doctoral study support from the China Scholarship Council (CSC) (CSC No. 201608080112) at Ludwig-Maximilians-University of Munich. Dr. Simin Li was funded by the Science Research Cultivation Program of Stomatological Hospital, Southern Medical University (Guangdong Provincial Stomatological Hospital) (No. PY2020004).

\section{Supplementary Materials}

Supplementary 1. File S1: the topological characteristics of all DEG nodes in the PPI network shown in Figure 4.

Supplementary 2. File S2: the topological characteristics of all nodes in the DElncRNA-DEG interaction network.

Supplementary 3. File S3: the topological characteristics of all nodes in the TF-DEG interaction network.

Supplementary 4. File S4: the topological characteristics of all DEG nodes in the PPI network for the significant blue module of GSE77459 dataset.

Supplementary 5. File S5: the topological characteristics of all DEG nodes in the PPI network for the significant green module of GSE92681 dataset.

Supplementary 6. File S6: the topological characteristics of all nodes in the ceRNA network.

\section{References}

[1] N. Jain, A. Gupta, and N. Meena, "An insight into neurophysiology of pulpal pain: facts and hypotheses," The Korean journal of pain, vol. 26, no. 4, pp. 347-355, 2013.

[2] P. R. Cooper, I. J. Chicca, M. J. Holder, and M. R. Milward, "Inflammation and regeneration in the dentin-pulp complex: net gain or net loss?," Journal of Endodontia, vol. 43, no. 9, pp. S87-s94, 2017.

[3] A. Agnihotry, W. Thompson, Z. Fedorowicz, E. J. van Zuuren, and J. Sprakel, "Antibiotic use for irreversible pulpitis," Cochrane Database Syst Rev, vol. 5, article Cd004969, no. 5, 2019.

[4] C. A. Murray and W. P. Saunders, "Root canal treatment and general health: a review of the literature," International Endodontic Journal, vol. 33, no. 1, pp. 1-18, 2000.

[5] S.-B. Yong, J. Y. Chung, Y. Song, and Y. H. Kim, "Recent challenges and advances in genetically-engineered cell therapy," Journal of Pharmaceutical Investigation, vol. 48, no. 2, pp. 199-208, 2018.

[6] X. Huang and K. Chen, "Differential expression of long noncoding RNAs in normal and inflamed human dental pulp," Journal of Endodontia, vol. 44, no. 1, pp. 62-72, 2018.

[7] J. L. McLachlan, A. J. Smith, I. J. Bujalska, and P. R. Cooper, "Gene expression profiling of pulpal tissue reveals the molecular complexity of dental caries," Biochimica et Biophysica Acta, vol. 1741, no. 3, pp. 271-281, 2005.

[8] X. Dong, W. Gao, L. V. Xiaoling et al., “Association between lncRNA GAS5, MEG3, and PCAT-1 polymorphisms and cancer risk: a meta-analysis," Disease Markers, vol. 2020, Article ID 6723487, 2020.

[9] W.-n. Hu, H.-x. Ding, Q. Xu, X.-y. Zhang, D.-t. Yang, and Y.z. Jin, "Relationship between long noncoding RNA H19 polymorphisms and risk of coronary artery disease in a Chinese population: a case-control study," Disease Markers, vol. 2020, Article ID 9839612, 2020.

[10] L. Salmena, L. Poliseno, Y. Tay, L. Kats, and P. P. Pandolfi, “A _ceRNA_Hypothesis: The Rosetta Stone of a Hidden RNA Language?," Cell, vol. 146, no. 3, pp. 353-358, 2011.

[11] Y. Yao, T. Zhang, L. Qi et al., "Competitive endogenous RNA network construction and comparison of lung squamous cell carcinoma in smokers and nonsmokers," Disease Markers, vol. 2019, Article ID 5292787, 2019.

[12] F. Lei, H. Zhang, and X. Xie, "Comprehensive analysis of an lncRNA-miRNA-mRNA competing endogenous RNA network in pulpitis," PeerJ, vol. 7, article e7135, 2019.

[13] E. Clough and T. Barrett, "The Gene Expression Omnibus database," Methods in Molecular Biology, vol. 1418, pp. 93$110,2016$.

[14] P. Sethupathy, B. Corda, and A. G. Hatzigeorgiou, "TarBase: a comprehensive database of experimentally supported animal microRNA targets," RNA, vol. 12, no. 2, pp. 192-197, 2006.

[15] S. D. Hsu, F. M. Lin, W. Y. Wu et al., "miRTarBase: a database curates experimentally validated microRNA-target interactions," Nucleic Acids Research, vol. 39, suppl_1, pp. D163D169, 2011.

[16] F. Xiao, Z. Zuo, G. Cai, S. Kang, X. Gao, and T. Li, "miRecords: an integrated resource for microRNA-target interactions," Nucleic Acids Research, vol. 37, no. Database, pp. D105D110, 2009. 
[17] J. H. Yang, J. H. Li, P. Shao, H. Zhou, Y. Q. Chen, and L. H. Qu, "starBase: a database for exploring microRNA-mRNA interaction maps from Argonaute CLIP-Seq and Degradome-Seq data," Nucleic Acids Research, vol. 39, suppl_1, pp. D202D209, 2011.

[18] M. E. Ritchie, B. Phipson, D. Wu et al., "limma powers differential expression analyses for RNA-sequencing and microarray studies," Nucleic Acids Research, vol. 43, no. 7, pp. e47e47, 2015.

[19] G. Yu, L. G. Wang, Y. Han, and Q. Y. He, “clusterProfiler: an R package for comparing biological themes among gene clusters," Omics: a journal of integrative biology, vol. 16, no. 5, pp. 284-287, 2012.

[20] S. Peri, "Human protein reference database as a discovery resource for proteomics," Nucleic Acids Research, vol. 32, no. 90001, pp. 497D-4501, 2004.

[21] R. Oughtred, C. Stark, B. J. Breitkreutz et al., “The BioGRID interaction database: 2019 update," Nucleic Acids Research, vol. 47, no. D1, pp. D529-d541, 2019.

[22] P. Shannon, A. Markiel, O. Ozier et al., "Cytoscape: a software environment for integrated models of biomolecular interaction networks," Genome Research, vol. 13, no. 11, pp. 24982504, 2003.

[23] D. Merico, R. Isserlin, O. Stueker, A. Emili, and G. D. Bader, "Enrichment map: a network-based method for gene-set enrichment visualization and interpretation," PLoS One, vol. 5, no. 11, article e13984, 2010.

[24] E. Wingender, P. Dietze, H. Karas, and R. Knüppel, “TRANSFAC: a database on transcription factors and their DNA binding sites," Nucleic Acids Research, vol. 24, no. 1, pp. 238-241, 1996.

[25] C. Jiang, Z. Xuan, F. Zhao, and M. Q. Zhang, "TRED: a transcriptional regulatory element database, new entries and other development," Nucleic Acids Research, vol. 35, no. Database, pp. D137-D140, 2007.

[26] F. Vafaee, J. R. Krycer, X. Ma, T. Burykin, D. E. James, and Z. Kuncic, "ORTI: an open-access repository of transcriptional interactions for interrogating mammalian gene expression data," PLoS One, vol. 11, no. 10, article e0164535, 2016.

[27] I. E. Wertz and V. M. Dixit, "Signaling to NF-kappaB: regulation by ubiquitination," Cold Spring Harbor Perspectives in Biology, vol. 2, no. 3, p. a003350, 2010.

[28] S. Raasi, G. Schmidtke, and M. Groettrup, "The ubiquitin-like protein FAT10 forms covalent conjugates and induces apoptosis," Journal of Biological Chemistry, vol. 276, no. 38, pp. 35334-35343, 2001.

[29] F. Ebstein, N. Lange, S. Urban, U. Seifert, E. Krüger, and P. M. Kloetzel, "Maturation of human dendritic cells is accompanied by functional remodelling of the ubiquitin-proteasome system," The International Journal of Biochemistry \& Cell Biology, vol. 41, no. 5, pp. 1205-1215, 2009.

[30] T. Yujiri, S. Sather, G. R. Fanger, and G. L. Johnson, "Role of MEKK1 in cell survival and activation of JNK and ERK pathways defined by targeted gene disruption," Science, vol. 282, no. 5395, pp. 1911-1914, 1998.

[31] F. S. Lee, J. Hagler, Z. J. Chen, and T. Maniatis, “Activation of the I $\kappa \mathrm{B} \alpha$ kinase complex by MEKK1, a kinase of the JNK pathway," Cell, vol. 88, no. 2, pp. 213-222, 1997.

[32] R. Al-Sadi, D. Ye, H. M. Said, and T. Y. Ma, "IL-1 $\beta$-induced increase in intestinal epithelial tight junction permeability is mediated by MEKK-1 activation of canonical NF- $\kappa$ B path- way," The American Journal of Pathology, vol. 177, no. 5, pp. 2310-2322, 2010.

[33] A. J. Resler, K. E. Malone, L. G. Johnson et al., "Genetic variation in TLR or NFkappaB pathways and the risk of breast cancer: a case-control study," BMC Cancer, vol. 13, no. 1, 2013.

[34] S. Sun, J. Sun, W. Jiang, W. Wang, and L. Ni, "Nav1. 7 via promotion of ERK in the trigeminal ganglion plays an important role in the induction of pulpitis inflammatory pain," BioMed Research International, vol. 2019, Article ID 6973932, 2019.

[35] J. Chang, C. Zhang, N. Tani-Ishii, S. Shi, and C. Y. Wang, "NF$\kappa \mathrm{B}$ activation in human dental pulp stem cells by TNF and LPS," Journal of Dental Research, vol. 84, no. 11, pp. 994998, 2005.

[36] A. M. Hashemi, S. S. Kahnamouii, H. Aghajani et al., "Quercetin decreases Th17 production by down-regulation of MAPKTLR4 signaling pathway on T cells in dental pulpitis," Journal of Dentistry, vol. 19, no. 4, p. 259, 2018.

[37] F. Cimmino, M. Avitabile, V. A. Lasorsa et al., "HIF-1 transcription activity: HIF1A driven response in normoxia and in hypoxia," BMC Medical Genetics, vol. 20, no. 1, p. 37, 2019.

[38] A. J. Majmundar, W. J. Wong, and M. C. Simon, "Hypoxiainducible factors and the response to hypoxic stress," Molecular Cell, vol. 40, no. 2, pp. 294-309, 2010.

[39] P. M. Dixon, N. Toitdu, and I. T. Dacre, CHAPTER 10- equine dental pathology, in Equine Dentistry (Third Edition), J. Easley, P. M. Dixon, and J. Schumacher, Eds., W.B. Saunders, Edinburgh, 2011.

[40] D. R. McIlwain, T. Berger, and T. W. Mak, "Caspase functions in cell death and disease," Cold Spring Harbor Perspectives in Biology, vol. 5, no. 4, article a008656, 2013.

[41] H. Qian, Q. Huang, Y.-X. Chen, Q. Liu, J.-X. Fang, and M.W. Ye, "Caspase-9 was involved in cell apoptosis in human dental pulp stem cells from deciduous teeth," Molecular Medicine Reports, vol. 18, no. 1, pp. 1067-1073, 2018.

[42] V. Viswanath, Y. Wu, R. Boonplueang et al., "Caspase-9 activation results in downstream caspase- 8 activation and bid cleavage in 1-methyl-4-phenyl-1, 2, 3, 6-tetrahydropyridineinduced Parkinson's disease," Journal of Neuroscience, vol. 21, no. 24, pp. 9519-9528, 2001.

[43] C.-L. Hahn, A. M. Best, and J. G. Tew, "Cytokine induction by Streptococcus mutans and pulpal pathogenesis," Infection and Immunity, vol. 68, no. 12, pp. 6785-6789, 2000.

[44] F. P. Cardoso, M. B. Viana, A. P. R. Sobrinho et al., "Methylation pattern of the _IFN_ $-\gamma$ gene in human dental pulp," Journal of Endodontics, vol. 36, no. 4, pp. 642-646, 2010.

[45] S. C. Chattipakorn, A. Sigurdsson, A. R. Light, M. Narhi, and W. Maixner, "Trigeminal c-Fos expression and behavioral responses to pulpal inflammation in ferrets," Pain, vol. 99, no. 1, pp. 61-69, 2002.

[46] M. R. Byers, E. H. Chudler, and M. J. Iadarola, "Chronic tooth pulp inflammation causes transient and persistent expression of Fos in dynorphin-rich regions of rat brainstem," Brain Research, vol. 861, no. 2, pp. 191-207, 2000.

[47] G. . H. Wabnitz, T. Köcher, P. Lohneis et al., "Costimulation induced phosphorylation of L-plastin facilitates surface transport of the T cell activation molecules CD69 and CD25," European Journal of Immunology, vol. 37, no. 3, pp. 649-662, 2007.

[48] M. Jontell, T. Okiji, U. Dahlgren, and G. Bergenholtz, "Immune defense mechanisms of the dental pulp," Critical Reviews in Oral Biology and Medicine, vol. 9, no. 2, pp. 179200, 1998. 
[49] R. Cao and Y. Zhang, "The functions of E(Z)/EZH2-mediated methylation of lysine 27 in histone H3," Current Opinion in Genetics \& Development, vol. 14, no. 2, pp. 155-164, 2004.

[50] T. Hui, P. A, Y. Zhao et al., "EZH2, a potential regulator of dental pulp inflammation and regeneration," Journal of Endodontia, vol. 40, no. 8, pp. 1132-1138, 2014.

[51] B. Li, F. Yu, F. Wu et al., "EZH2 impairs human dental pulp cell mineralization via the Wnt/ $\beta$-catenin pathway," Journal of Dental Research, vol. 97, no. 5, pp. 571-579, 2018.

[52] Q. I. HUANG, J. U. N. L. I. XUE, R. U. N. M. E. I. ZOU et al., "NR4A1 is associated with chronic low-grade inflammation in patients with type 2 diabetes," Experimental and Therapeutic Medicine, vol. 8, no. 5, pp. 1648-1654, 2014.

[53] N. Ipseiz, S. Uderhardt, C. Scholtysek et al., "The nuclear receptor Nr4a1 mediates anti-inflammatory effects of apoptotic cells," Journal of Immunology, vol. 192, no. 10, pp. 48524858, 2014.

[54] H. Dweep, C. Sticht, P. Pandey, and N. Gretz, "miRWalk database: prediction of possible miRNA binding sites by "walking" the genes of three genomes," Journal of Biomedical Informatics, vol. 44, no. 5, pp. 839-847, 2011.

[55] Y. Li, C. Dai, C. Wu, and Y. Liu, "PINCH-1 promotes tubular epithelial-to-mesenchymal transition by interacting with integrin-linked kinase," J Am Soc Nephrol, vol. 18, no. 9, pp. 2534-2543, 2007.

[56] S.-Y. Lee, H. M. Yun, R. A. Perez et al., "Nanotopological-tailored calcium phosphate cements for the odontogenic stimulation of human dental pulp stem cells through integrin signaling," RSC Advances, vol. 5, no. 78, pp. 63363-63371, 2015.

[57] D. De Nardo, K. R. Balka, Y. C. Gloria, V. R. Rao, E. Latz, and S. L. Masters, "Interleukin-1 receptor-associated kinase 4 (IRAK4) plays a dual role in myddosome formation and Toll-like receptor signaling," The Journal of Biological Chemistry, vol. 293, no. 39, pp. 15195-15207, 2018.

[58] Q. Zhou, R. Gallagher, R. Ufret-Vincenty, X. Li, E. N. Olson, and S. Wang, "Regulation of angiogenesis and choroidal neovascularization by members of microRNA-23 27 24 clusters," Proceedings of the National Academy of Sciences of the United States of America, vol. 108, no. 20, pp. 8287-8292, 2011.

[59] K. Takahashi, "Pulpal vascular changes in inflammation," Proc Finn Dent Soc, 1992, Suppl 1:381-5.

[60] A. Hindley and W. Kolch, "Extracellular signal regulated kinase (ERK)/mitogen activated protein kinase (MAPK)-independent functions of Raf kinases," Journal of Cell Science, vol. 115, Part 8, pp. 1575-1581, 2002.

[61] S. Simon, A. J. Smith, A. Berdal, P. J. Lumley, and P. R. Cooper, "The MAP kinase pathway is involved in odontoblast stimulation via p38 phosphorylation," Journal of Endodontia, vol. 36, no. 2, pp. 256-259, 2010.

[62] S. Zhong, S. Zhang, E. Bair, S. Nares, and A. A. Khan, "Differential expression of microRNAs in normal and inflamed human pulps," Journal of Endodontia, vol. 38, no. 6, pp. 746752, 2012.

[63] B. E. Dayanc, S. Bansal, A. O. Gure, S. O. Gollnick, and E. A. Repasky, "Enhanced sensitivity of colon tumour cells to natural killer cell cytotoxicity after mild thermal stress is regulated through HSF1-mediated expression of MICA," International Journal of Hyperthermia, vol. 29, no. 5, pp. 480490, 2013.
[64] I. Z. Oskui, M. N. Ashtiani, A. Hashemi, and H. Jafarzadeh, "Effect of thermal stresses on the mechanism of tooth pain," Journal of Endodontia, vol. 40, no. 11, pp. 1835-1839, 2014.

[65] D. B. Pontier and J. Gribnau, "Xist regulation and function explored," Human Genetics, vol. 130, no. 2, pp. 223-236, 2011.

[66] X. Wu, X. Dinglin, X. Wang et al., "Long noncoding RNA XIST promotes malignancies of esophageal squamous cell carcinoma via regulation of miR-101/EZH2," Oncotarget, vol. 8, no. 44, pp. 76015-76028, 2017.

[67] Q. Zhou, W. Hu, W. Zhu et al., "Long non coding RNA XIST as a prognostic cancer marker - a meta-analysis," Clinica Chimica Acta, vol. 482, pp. 1-7, 2018.

[68] L. Ma, Y. Zhou, X. Luo, H. Gao, X. Deng, and Y. Jiang, "Long non-coding RNA XIST promotes cell growth and invasion through regulating miR-497/MACC1 axis in gastric cancer," Oncotarget, vol. 8, no. 3, pp. 4125-4135, 2017.

[69] B. Shenoda, Y. Tian, G. Alexander, E. Aradillas-Lopez, R. Schwartzman, and S. Ajit, "miR-34a-mediated regulation of XIST in female cells under inflammation," Journal of Pain Research, vol. Volume 11, pp. 935-945, 2018.

[70] M. Ma, Y. Pei, X. Wang, J. Feng, Y. Zhang, and M. Q. Gao, "LncRNA XIST mediates bovine mammary epithelial cell inflammatory response via NF- $\kappa \mathrm{B} / \mathrm{NLRP} 3$ inflammasome pathway," Cell Proliferation, vol. 52, no. 1, article e12525, 2019.

[71] K. Vargova, N. Curik, P. Burda et al., "MYB transcriptionally regulates the miR-155 host gene in chronic lymphocytic leukemia," Blood, vol. 117, no. 14, pp. 3816-3825, 2011.

[72] E. Vigorito, S. Kohlhaas, D. Lu, and R. Leyland, "miR-155: an ancient regulator of the immune system," Immunological Reviews, vol. 253, no. 1, pp. 146-157, 2013.

[73] X. Wu, Y. Wang, T. Yu et al., "Blocking MIR155HG/miR-155 axis inhibits mesenchymal transition in glioma," Neuro-Oncology, vol. 19, no. 9, pp. 1195-1205, 2017.

[74] G. Dodt and S. J. Gould, "Multiple PEX genes are required for proper subcellular distribution and stability of Pex5p, the PTS1 receptor: evidence that PTS1 protein import is mediated by a cycling receptor," The Journal of Cell Biology, vol. 135, no. 6, pp. 1763-1774, 1996.

[75] F. Di Cara, A. Sheshachalam, N. E. Braverman, R. A. Rachubinski, and A. J. Simmonds, "Peroxisome-mediated metabolism is required for immune response to microbial infection," Immunity, vol. 48, no. 4, pp. 832-833, 2018.

[76] D. M. Dorfman, D. B. Wilson, G. A. Bruns, and S. H. Orkin, "Human transcription factor GATA-2. Evidence for regulation of preproendothelin-1 gene expression in endothelial cells.," The Journal of Biological Chemistry, vol. 267, no. 2, pp. 1279-1285, 1992.

[77] M. Liu, L. Zhao, J. Hu et al., "Endothelial cells and endothelin-1 promote the odontogenic differentiation of dental pulp stem cells," Molecular Medicine Reports, vol. 18, no. 1, pp. 893-901, 2018.

[78] R. P. Panganiban, B. M. Vonakis, F. T. Ishmael, and C. Stellato, "Coordinated post-transcriptional regulation of the chemokine system: messages from CCL2," Journal of Interferon \& Cytokine Research, vol. 34, no. 4, pp. 255-266, 2014.

[79] J. S. Yordy, O. Moussa, H. Pei, D. Chaussabel, R. Li, and D. K. Watson, "SP100 inhibits ETS1 activity in primary endothelial cells," Oncogene, vol. 24, no. 5, pp. 916-931, 2005.

[80] W. Chen, W. Jin, N. Hardegen et al., "Conversion of peripheral CD4+CD25- naive $\mathrm{T}$ cells to $\mathrm{CD} 4+\mathrm{CD} 25+$ regulatory $\mathrm{T}$ cells by TGF-beta induction of transcription factor Foxp3," The 
Journal of Experimental Medicine, vol. 198, no. 12, pp. 18751886, 2003.

[81] Y. Zheng and A. Y. Rudensky, "Foxp3 in control of the regulatory T cell lineage," Nature Immunology, vol. 8, no. 5, pp. 457462, 2007.

[82] K. F. Bruno, J. A. Silva, T. A. Silva, A. C. Batista, A. H. G. Alencar, and C. Estrela, "Characterization of inflammatory cell infiltrate in human dental pulpitis," International Endodontic Journal, vol. 43, no. 11, pp. 1013-1021, 2010.

[83] D. Bayarsaihan, "Epigenetic mechanisms in inflammation," Journal of Dental Research, vol. 90, no. 1, pp. 9-17, 2010.

[84] S. Sonoda, H. Yamaza, L. Ma et al., "Interferon-gamma improves impaired dentinogenic and immunosuppressive functions of irreversible pulpitis-derived human dental pulp stem cells," Scientific Reports, vol. 6, no. 1, 2016.

[85] M. Elsalhy, F. Azizieh, and R. Raghupathy, "Cytokines as diagnostic markers of pulpal inflammation," International Endodontic Journal, vol. 46, no. 6, pp. 573-580, 2013.

[86] M. Zehnder, N. Delaleu, Y. du, and M. Bickel, "Cytokine gene expression-part of host defence in pulpitis," Cytokine, vol. 22, no. 3-4, pp. 84-88, 2003.

[87] K. Derringer and R. Linden, "Epidermal growth factor released in human dental pulp following orthodontic force," European Journal of Orthodontics, vol. 29, no. 1, pp. 67-71, 2007.

[88] M. Zhang, F. Jiang, X. Zhang et al., "The effects of plateletderived growth factor-BB on human dental pulp stem cells mediated dentin-pulp complex regeneration," Stem Cells Translational Medicine, vol. 6, no. 12, pp. 2126-2134, 2017.

[89] Y. Zhang, X. H. Feng, and R. Derynck, "Smad3 and Smad4 cooperate with c-Jun/c-Fos to mediate TGF- $\beta$-induced transcription," Nature, vol. 394, no. 6696, pp. 909-913, 1998.

[90] T. Haniastuti, P. Nunez, and A. Djais, "The role of transforming growth factor beta in tertiary dentinogenesis," Dental Journal (Majalah Kedokteran Gigi), vol. 41, 2015.

[91] J.-H. Jang, H. W. Shin, J. M. Lee, H. W. Lee, E. C. Kim, and S. H. Park, "An overview of pathogen recognition receptors for innate immunity in dental pulp," Mediators of Inflammation, vol. 2015, 12 pages, 2015.

[92] N. S. T. Hozhabri, M. D. Benson, M. D. Vu et al., "Decreasing NF- $\kappa$ B expression enhances odontoblastic differentiation and collagen expression in dental pulp stem cells exposed to inflammatory cytokines," PLoS One, vol. 10, no. 1, article e0113334, 2015.

[93] A. B. Kokkas, A. Goulas, K. Varsamidis, V. Mirtsou, and D. Tziafas, "Irreversible but not reversible pulpitis is associated with up-regulation of tumour necrosis factor-alpha gene expression in human pulp," International Endodontic Journal, vol. 40, no. 3, pp. 198-203, 2007.

[94] B. E. Hall, L. Zhang, Z. J. Sun et al., "Conditional TNF- $\alpha$ overexpression in the tooth and alveolar bone results in painful pulpitis and osteitis," Journal of Dental Research, vol. 95, no. 2, pp. 188-195, 2016.

[95] Y. Sawa, S. Yoshida, K. I. Shibata, M. Suzuki, and A. Mukaida, "Vascular endothelium of human dental pulp expresses diverse adhesion molecules for leukocyte emigration," Tissue and Cell, vol. 30, no. 2, pp. 281-291, 1998.

[96] H. Yumoto, K. Hirao, Y. Hosokawa et al., "The roles of odontoblasts in dental pulp innate immunity," Japanese Dental Science Review, vol. 54, no. 3, pp. 105-117, 2018. 